\title{
Output-Based Error Estimation and Mesh Adaptation in Computational Fluid Dynamics: Overview and Recent Results
}

\author{
Krzysztof J. Fidkowski * \\ University of Michigan, Ann Arbor, MI 48109 \\ David L. Darmofal ${ }^{\dagger}$ \\ Massachusetts Institute of Technology, Cambridge, MA 02139
}

This article reviews recent work in output-based error estimation and mesh adaptation for Computational Fluid Dynamics (CFD) applications in aerospace engineering. Output adjoint solutions, which play a central role in the error estimation, are introduced both in fully-discrete and in variational formulations. The adjoint-weighted residual method for error estimation is then presented in both formulations. Assumptions and approximations made in the calculations are discussed. Presentation of the discrete and variational formulations enables a side-by-side comparison of recent work in output error estimation using the finite volume method and the finite element method. Various mesh adaptation techniques for reducing output error are then discussed. Recent adaptive results using different implementations are presented for a variety of applications. These results show the power of output-based adaptive methods for improving the robustness of CFD computations. However, challenges and areas of additional future research, such as computable error bounds and robust mesh adaptation mechanics, exist, and these are discussed following the results.

\footnotetext{
*Assistant Professor, Department of Aerospace Engineering, 3029 François-Xavier Bagnoud Building, Member AIAA.

${ }^{\dagger}$ Associate Department Head / Associate Professor, Department of Aerospace Engineering, Building 33, Room 207. Senior Member AIAA. 


\section{Contents}

I Introduction $\quad 3$

II Outputs and Adjoints $\quad 5$

II.A Fully-Discrete Formulation . . . . . . . . . . . . . . . . . . . 5

II.B Variational Formulation . . . . . . . . . . . . . . . . . . . 5

II.C Adjoint Consistency . . . . . . . . . . . . . . . . . . . 6

II.D Mean-Value Linearization . . . . . . . . . . . . . . . . . . 7

II.E Adjoint Implementation $\ldots \ldots \ldots \ldots$

$\begin{array}{lr}\text { III Error Estimation } & 9\end{array}$

III.A Forms of Error Estimation . . . . . . . . . . . . . . . . . . 9

III.B The Adjoint-Weighted Residual Method . . . . . . . . . . . . . . . . . . 10

III.C Fully-Discrete Formulation . . . . . . . . . . . . . . . . . . . . . . 11

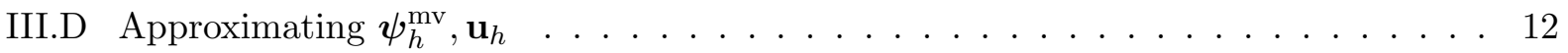

III.E Computable Corrections and Remaining Error . . . . . . . . . . . . . . . . . 12

III.F Error Effectivity . . . . . . . . . . . . . . . . . . . . . . . . 13

III.G Impact of Shocks . . . . . . . . . . . . . . . . . . . . . . 13

IV Mesh Adaptation $\quad 14$

IV.A Error Localization . . . . . . . . . . . . . . . . . . . . . 14

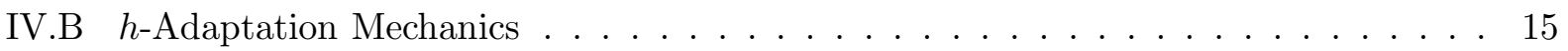

IV.C Overview of Adaptation Strategies . . . . . . . . . . . . . . . 16

IV.D Incorporating a priori Analysis and Anisotropy . . . . . . . . . . . . . 18

IV.E Direct Optimization Adaptation . . . . . . . . . . . . . . . . . 20

IV.F Cut-Cell Methods . . . . . . . . . . . . . . . . . . 20

V Implementations and Results $\quad 21$

V.A Finite Volume Methods . . . . . . . . . . . . . . . . . . . 21

V.B Discontinuous Galerkin Methods . . . . . . . . . . . . . . . . . 26

VI Challenges and Ongoing Research $\quad 31$

VI.A Robust Mesh Adaptation . . . . . . . . . . . . . . . . . 31

VI.B Computable Error Bounds . . . . . . . . . . . . . . . . . . . 32

VI.C Unsteady Applications . . . . . . . . . . . . . . . . . . . . 33

$\begin{array}{ll}\text { VII Acknowledgments } & 33\end{array}$ 


\section{Introduction}

The accessibility, fast turnaround time, and almost arbitrary test conditions offered by Computational Fluid Dynamics (CFD) make it an attractive tool in industry. CFD simulations with sophisticated physical modeling are now used regularly to reduce design cycle costs and to improve final product design. This prevalence of CFD has been driven by increasing computational power and improvements in numerical methods, to the extent that complex simulations on general three-dimensional geometries are now routine.

Such powerful simulation capability is a remarkable achievement for CFD, but it also comes with a new liability: ensuring that the solutions computed are sufficiently accurate. Typically, this liability is managed by practitioners knowledgeable about the assumptions and limitations of the models and discretization. However, even very experienced practitioners cannot quantify the error in a discrete approximation of a complex flowfield. In addition, reliance on best-practice guidelines for mesh generation and on previous experience is an open-loop solution that leaves the door open to large amounts of numerical error for computations on novel configurations.

Even on relatively standard simulations, questions arise regarding the robustness with which CFD methods can accurately compute outputs of interest. An example is the American Institute of Aeronautics and Astronautics Drag Prediction Workshop (DPW), ${ }^{1-3}$ in which force and moment outputs for a representative set of wing-body geometries and flow conditions were compared across codes in industry and academia. Results from submissions have consistently shown a wide degree of scatter in computed outputs. For example, drag coefficient variations of .0025 observed in the most recent DPW on a DLR-F6 wing body (Figure 1) translate to a difference of over 100 passengers on a large transport aircraft. ${ }^{4,5}$ The results from the most recent workshop constitute only a slight

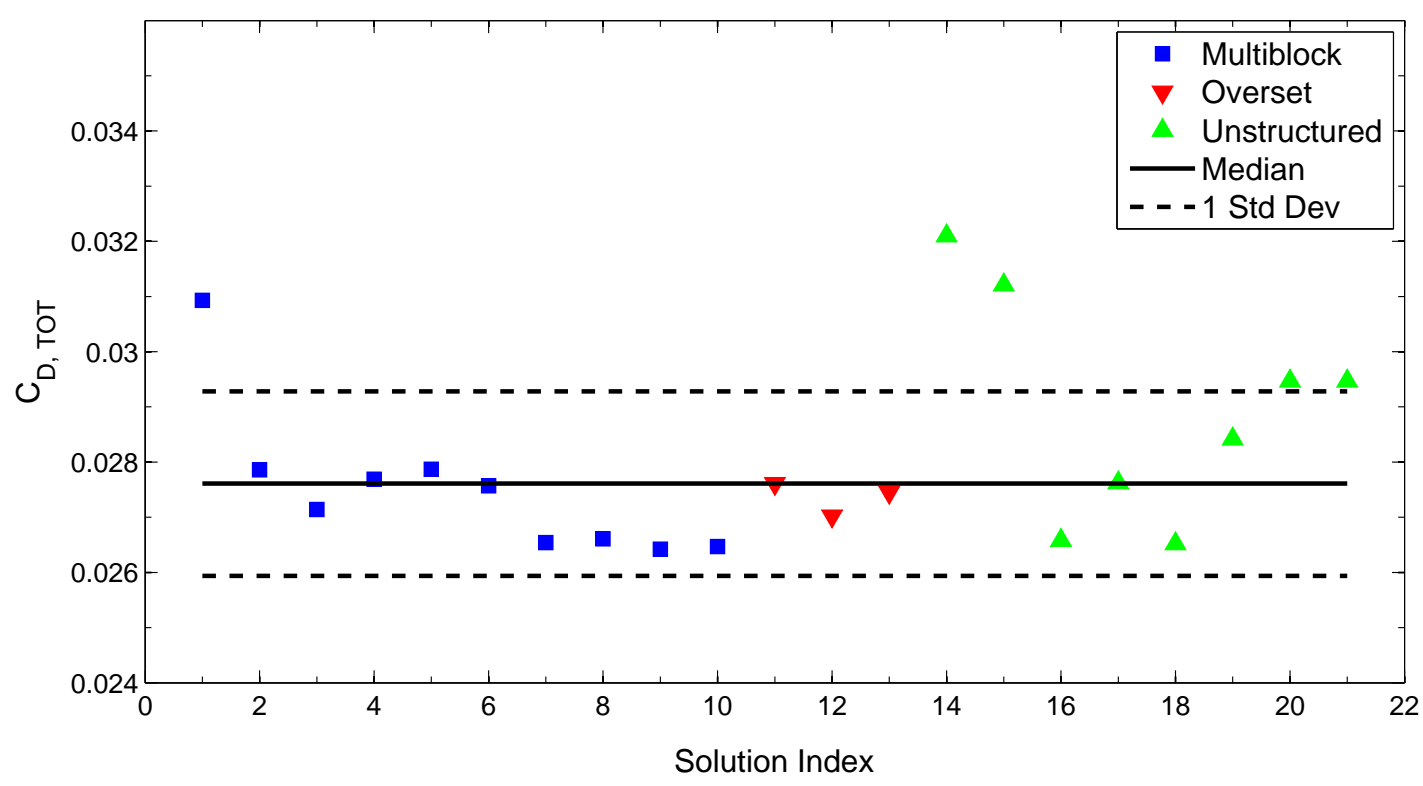

Figure 1. DPW III results: total drag coefficient predictions for the DLR-F6 wing-body at $M=0.75, C_{L}=0.5, R e=5 \times 10^{6}$. The solution index differentiates between different codes, turbulence models, and mesh types..$^{3,6}$ 
improvement over the results from the two previous workshops, ${ }^{1,2}$ even though computational power has increased substantially. Moreover, additional tests in the recent DPW show that discrepancies persist even for simple wing-only geometries. For these geometries, results obtained using the same code but on independently-generated meshes appear to converge to different output values with uniform mesh refinement. ${ }^{7}$ This lack of grid convergence, illustrated in Figure 2, indicates that the highly-disparate length scales in this three dimensional flow are not adequately resolved even with some of the finest a priori generated meshes. This observation suggests that increases in
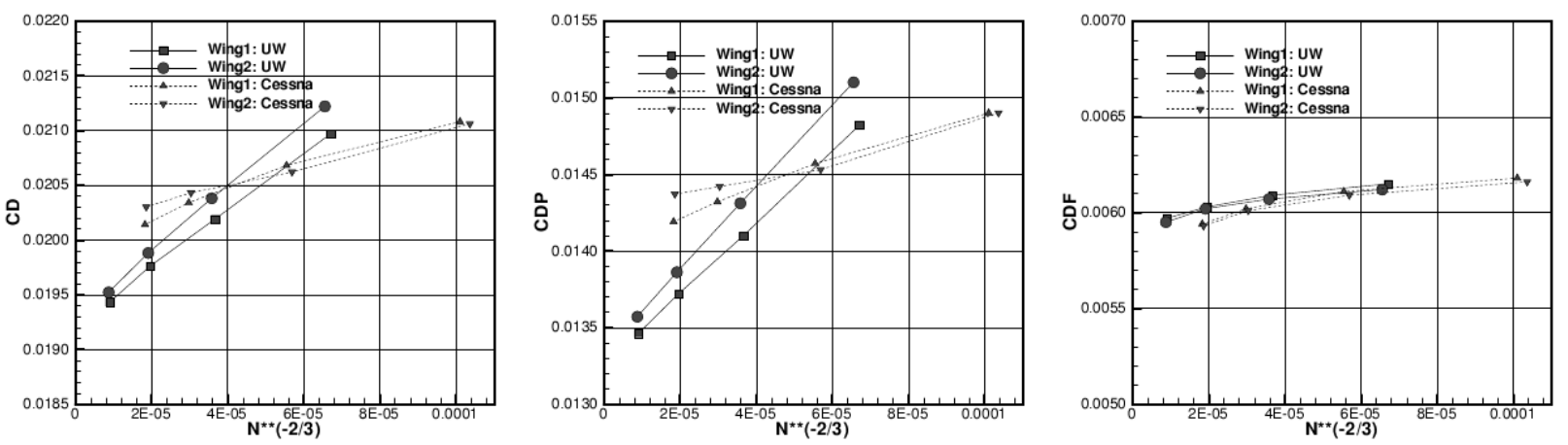

Figure 2. DPW III wing-only results: total, pressure and skin friction drag convergence for two families of grids of two wing geometries, at $M=0.76, \alpha=0.5^{\circ}, R e=5 \times 10^{6}$. One set of grids was generated by Cessna Aircraft Co. and the other by the University of Wyoming. Reproduced with permission from. ${ }^{7}$

computational power alone will be insufficient to decrease numerical error for such applications to acceptable levels in the near future.

For each answer in Figure 1, an additional useful piece of information would be an associated confidence measure, for example in the form of an error bar. Providing such error bars is the goal of output-based error estimation techniques. The general idea of error estimation is not a new concept, and a number of previous works have reviewed the subject in various contexts. Verfurth analyzes a posteriori error estimates for elliptic partial differential equations, and shows an equivalence between estimates based on local residuals and on solutions of local problems. ${ }^{8}$ Ainsworth and Oden focus on mechanics and consider a posteriori energy norm error estimates for linear elliptic boundary value problems. ${ }^{9}$ In the context of output error estimation, Becker and Rannacher present a thorough review of the adjoint-weighted residual method for a posteriori error estimation in finite element discretizations of elliptic, parabolic, and hyperbolic equations. ${ }^{10}$ In addition, Giles and Pierce review adjoint correction techniques and Giles and Süli review a posteriori output error estimation for finite element methods applied to linear and nonlinear partial differential equations relevant to CFD. ${ }^{11,12}$

This paper reviews output error estimation and mesh adaptation specifically in the context of aerospace CFD applications and it presents a collection of recent results for practical problems. The structure of the paper is as follows. Section II introduces output adjoint solutions for both fullydiscrete and variational problems. Section III then reviews the adjoint-weighted residual method for output-based error estimation. Error localization and mesh adaptation techniques are reviewed in Section IV. Section V presents recent implementations and results for aerospace engineering applications. Finally, challenges and ongoing research are discussed in Section VI. 


\section{Outputs and Adjoints}

\section{II.A. Fully-Discrete Formulation}

Consider a partial differential equation discretized into $N_{h}$, possibly nonlinear, algebraic equations,

$$
\mathbf{R}_{h}\left(\mathbf{u}_{h}\right)=0
$$

where $\mathbf{u}_{h} \in \mathbb{R}^{N_{h}}$ is the vector of unknowns and $\mathbf{R}_{h} \in \mathbb{R}^{N_{h}}$ is the vector of residuals that must be driven to zero. The subscript $h$ denotes the fineness of the discretization and encompasses both mesh size and interpolation order. Given a scalar output $J_{h}\left(\mathbf{u}_{h}\right)$, the associated adjoint vector, $\boldsymbol{\psi}_{h} \in \mathbb{R}^{N_{h}}$, is the sensitivity of $J_{h}$ to an infinitesimal residual perturbation, $\delta \mathbf{R}_{h} \in \mathbb{R}^{N_{h}}$, to the nonlinear system:

$$
\delta J_{h} \equiv J_{h}\left(\mathbf{u}_{h}+\delta \mathbf{u}_{h}\right)-J_{h}\left(\mathbf{u}_{h}\right) \equiv \boldsymbol{\psi}_{h}^{T} \delta \mathbf{R}_{h}
$$

where $\delta \mathbf{u}_{h}$ is the infinitesimal solution perturbation satisfying

$$
\frac{\partial \mathbf{R}_{h}}{\partial \mathbf{u}_{h}} \delta \mathbf{u}_{h}+\delta \mathbf{R}_{h}=0
$$

which is obtained by linearizing Eqn. 1. The linearization assumes the discrete equations are differentiable. Further, assuming that the output is also differentiable,

$$
\delta J_{h}=\frac{\partial J_{h}}{\partial \mathbf{u}_{h}} \delta \mathbf{u}_{h}=-\boldsymbol{\psi}_{h}^{T} \frac{\partial \mathbf{R}_{h}}{\partial \mathbf{u}_{h}} \delta \mathbf{u}_{h}
$$

where Eqns. 2 and 3 were used in the second equality. In order for Eqn. 4 to hold for arbitrary perturbations, $\psi_{h}$ must satisfy the discrete adjoint equation

$$
\left(\frac{\partial \mathbf{R}_{h}}{\partial \mathbf{u}_{h}}\right)^{T} \boldsymbol{\psi}_{h}+\left(\frac{\partial J_{h}}{\partial \mathbf{u}_{h}}\right)^{T}=0
$$

\section{II.B. Variational Formulation}

In a variational setting, consider a general semilinear form arising from a Galerkin weighted residual statement: determine $\mathbf{u}_{h} \in \mathcal{V}_{h}$ such that

$$
\mathcal{R}_{h}\left(\mathbf{u}_{h}, \mathbf{v}_{h}\right)=0, \quad \forall \mathbf{v}_{h} \in \mathcal{V}_{h},
$$

where $\mathcal{V}_{h}$ is a finite-dimensional space of functions. The subscript $h$ indicates a discretization of the computational domain, such as a triangulation in a finite element method. $\mathcal{R}_{h}(\cdot, \cdot): \mathcal{V}_{h} \times \mathcal{V}_{h} \rightarrow \mathbb{R}$ is assumed to be a semilinear form, linear in the second argument. A scalar output of interest is denoted by $\mathcal{J}_{h}(\cdot): \mathcal{V}_{h} \rightarrow \mathbb{R}$, where the subscript $h$ is included because the output calculation may involve discretization-dependent terms.

Consider an infinitesimal residual perturbation $\delta \mathbf{r}_{h} \in \mathcal{V}_{h}$ that creates a solution perturbation $\delta \mathbf{u}_{h} \in \mathcal{V}_{h}$ related via the statement:

$$
\mathcal{R}_{h}\left(\mathbf{u}_{h}+\delta \mathbf{u}_{h}, \mathbf{v}_{h}\right)+\left(\delta \mathbf{r}_{h}, \mathbf{v}_{h}\right)=0, \quad \forall \mathbf{v}_{h} \in \mathcal{V}_{h}
$$


where $(\cdot, \cdot): \mathcal{V}_{h} \times \mathcal{V}_{h} \rightarrow \mathbb{R}$ is a suitable inner product associated with the semilinear form. For this variational formulation, an adjoint $\boldsymbol{\psi}_{h} \in \mathcal{V}_{h}$ can be defined as the sensitivity of the output to the residual perturbation,

$$
\mathcal{J}_{h}\left(\mathbf{u}_{h}+\delta \mathbf{u}_{h}\right)-\mathcal{J}_{h}\left(\mathbf{u}_{h}\right)=\left(\delta \mathbf{r}_{h}, \boldsymbol{\psi}_{h}\right) .
$$

For infinitesimal perturbations, Fréchét linearizations about $\mathbf{u}_{h}$ are introduced in Eqns. 7 and 8 , resulting in

$$
\left(\delta \mathbf{r}_{h}, \boldsymbol{\psi}_{h}\right)=\mathcal{J}_{h}^{\prime}\left[\mathbf{u}_{h}\right]\left(\delta \mathbf{u}_{h}\right)=-\mathcal{R}_{h}^{\prime}\left[\mathbf{u}_{h}\right]\left(\delta \mathbf{u}_{h}, \boldsymbol{\psi}_{h}\right),
$$

where the prime denotes a Fréchét linearization with respect to the arguments in the square brackets. For these linearizations to exist, both the semilinear form and the output are assumed to be differentiable. In order for Eqn. 9 to be true for general perturbations, the adjoint must satisfy the statement: find $\boldsymbol{\psi}_{h} \in \mathcal{V}_{h}$ such that

$$
\mathcal{R}_{h}^{\prime}\left[\mathbf{u}_{h}\right]\left(\mathbf{v}_{h}, \boldsymbol{\psi}_{h}\right)+\mathcal{J}_{h}^{\prime}\left[\mathbf{u}_{h}\right]\left(\mathbf{v}_{h}\right)=0, \quad \forall \mathbf{v}_{h} \in \mathcal{V}_{h}
$$

Once a basis is chosen for the weighted residual statements, Eqns. 6 and 10 are equivalent to their discrete counterparts, Eqns. 1 and 5, respectively. While the fully-discrete formulation is simpler from an implementation perspective, the weighted-residual formulation offers more rigor for error estimation.

\section{II.C. Adjoint Consistency}

Eqns. 5 and 10 yield the discrete adjoint $\boldsymbol{\psi}_{h}$, either as a vector or as a function in a finite-dimensional space. Of interest is how this discrete solution compares to its continuous counterpart. Given the continuous primal solution, $\mathbf{u} \in \mathcal{V}$, satisfying

$$
\mathcal{R}(\mathbf{u}, \mathbf{v})=0, \quad \forall \mathbf{v} \in \mathcal{V},
$$

for an appropriately defined space $\mathcal{V}$, a continuous adjoint $\boldsymbol{\psi} \in \mathcal{V}$ satisfies

$$
\mathcal{R}^{\prime}[\mathbf{u}](\mathbf{v}, \boldsymbol{\psi})+\mathcal{J}^{\prime}[\mathbf{u}](\mathbf{v})=0, \quad \forall \mathbf{v} \in \mathcal{V} .
$$

For simplicity, we have assumed that both $\mathbf{u}$ and $\boldsymbol{\psi}$ are in $\mathcal{V}$. However, the space for the adjoint solution does not have to be the same as the space for the primal solution. ${ }^{13}$

The continuous adjoint can be regarded as a Green's function relating source perturbations in the original partial differential equation to perturbations in the output. To demonstrate this interpretation, a sample adjoint solution is illustrated in Figure 3 for subsonic flow over a lifting airfoil. Upstream of the airfoil, the adjoint is seen to vary rapidly across the stagnation streamline. This behavior was suggested in the analysis of Giles and Pierce who found that a square root singularity with respect to distance from the stagnation streamline exists for sources that perturb the stagnation pressure. ${ }^{14}$

To be precise, the adjoint field in Figure 3 is the discrete adjoint solution on a fine mesh. It can only be regarded as a faithful representation of the continuous adjoint if the discretization is in some manner consistent with the continuous adjoint problem. Primal consistency in the variational problem requires that the continuous solution $\mathbf{u}$ satisfies the discrete variational statement,

$$
\mathcal{R}_{h}(\mathbf{u}, \mathbf{v})=0, \quad \forall \mathbf{v} \in \mathcal{W}_{h},
$$




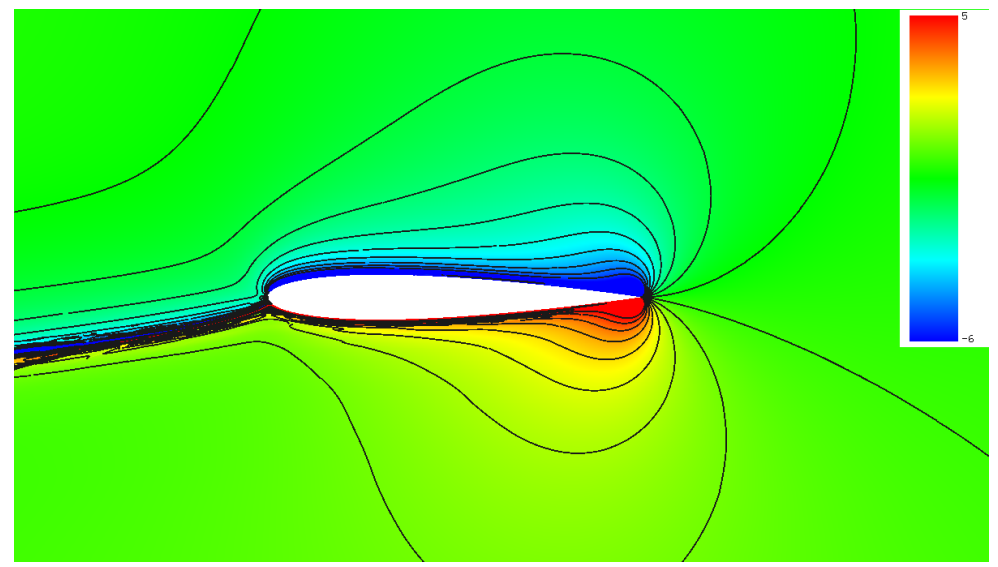

Figure 3. $x$-momentum component of the lift adjoint for a NACA 0012 airfoil at $M=0.4, \alpha=$ $5^{\circ}$. The continuous interpretation states that a residual perturbation removing $x$-momentum from the flow above the airfoil will tend to decrease the lift, whereas the opposite will be observed below the airfoil. Note, the sign of the adjoint depends on whether the source is added to the left or the right side of the original equation.

where $\mathcal{W}_{h}=\mathcal{V}+\mathcal{V}_{h}=\left\{h=f+g: f \in \mathcal{V}_{h}, g \in \mathcal{V}\right\}$. Similarly, the combination of the discrete semi-linear form $\mathcal{R}_{h}$ and the functional $\mathcal{J}_{h}$ is said to be adjoint consistent if ${ }^{13,15,16}$

$$
\mathcal{R}_{h}^{\prime}[\mathbf{u}](\mathbf{v}, \boldsymbol{\psi})+\mathcal{J}_{h}^{\prime}[\mathbf{u}](\mathbf{v})=0, \quad \forall \mathbf{v} \in \mathcal{W}_{h},
$$

Discretizations that are not adjoint consistent may still be asymptotically adjoint consistent if Eqn. 14 holds in the limit $h \rightarrow 0$, over suitably normalized $\mathbf{v} \in \mathcal{W}_{h}$.

Adjoint consistency has an impact on adjoint-based methods, including shape optimization, ${ }^{10,12,13}$ and on the convergence of the primal problem itself. ${ }^{17}$ In error estimation, an adjoint-inconsistent discretization can lead to irregular or oscillatory adjoint solutions that pollute the error estimate with noise and lead to adaptation in incorrect areas. Enforcing adjoint consistency imposes restrictions on the interior and boundary discretizations that enter into the semi-linear form, as well as on the output definition. These restrictions have been studied by several authors in the context of the discontinuous Galerkin method. ${ }^{13,15,17}$ In general, discretizations that are found to be adjoint inconsistent can often be made adjoint consistent by adding terms to either the semi-linear form or the output functional.

\section{II.D. Mean-Value Linearization}

Non-infinitesimal perturbations $\delta \mathbf{u}_{h}$ can be considered for non-linear problems if a mean-value linearization is used to define the adjoint. ${ }^{10,18-20}$

\section{II.D.1. Fully-discrete formulation}

The discrete mean-value adjoint, $\boldsymbol{\psi}_{h}^{\mathrm{mv}} \in \mathbb{R}^{N_{h}}$ satisfies

$$
\left(\overline{\mathbf{R}}_{h}\left[\mathbf{u}_{h}, \mathbf{u}_{h}+\delta \mathbf{u}_{h}\right]\right)^{T} \boldsymbol{\psi}_{h}^{\mathrm{mv}}+\left(\bar{J}_{h}\left[\mathbf{u}_{h}, \mathbf{u}_{h}+\delta \mathbf{u}_{h}\right]\right)^{T}=0
$$


where $\overline{\mathbf{R}}_{h} \in \mathbb{R}^{N_{h} \times N_{h}}$ and $\bar{J}_{h} \in \mathbb{R}^{N_{h}}$ satisfy

$$
\begin{aligned}
\overline{\mathbf{R}}_{h}\left[\mathbf{u}_{h}, \mathbf{u}_{h}+\delta \mathbf{u}_{h}\right] & =\int_{0}^{1} \frac{\partial \mathbf{R}_{h}}{\partial \mathbf{u}_{h}}\left[\mathbf{u}_{h}+\theta \delta \mathbf{u}_{h}\right] d \theta \\
\bar{J}_{h}\left[\mathbf{u}_{h}, \mathbf{u}_{h}+\delta \mathbf{u}_{h}\right] & =\int_{0}^{1} \frac{\partial J_{h}}{\partial \mathbf{u}_{h}}\left[\mathbf{u}_{h}+\theta \delta \mathbf{u}_{h}\right] d \theta
\end{aligned}
$$

Since

$$
\begin{aligned}
\overline{\mathbf{R}}_{h}\left[\mathbf{u}_{h}, \mathbf{u}_{h}+\delta \mathbf{u}_{h}\right] \delta \mathbf{u}_{h} & =\mathbf{R}_{h}\left(\mathbf{u}_{h}+\delta \mathbf{u}_{h}\right)-\mathbf{R}_{h}\left(\mathbf{u}_{h}\right), \\
\bar{J}_{h}\left[\mathbf{u}_{h}, \mathbf{u}_{h}+\delta \mathbf{u}_{h}\right] \delta \mathbf{u}_{h} & =J_{h}\left(\mathbf{u}_{h}+\delta \mathbf{u}_{h}\right)-J_{h}\left(\mathbf{u}_{h}\right),
\end{aligned}
$$

the output perturbation can be expressed as,

$$
\begin{aligned}
J_{h}\left(\mathbf{u}_{h}+\delta \mathbf{u}_{h}\right)-J_{h}\left(\mathbf{u}_{h}\right) & =\bar{J}_{h}\left[\mathbf{u}_{h}, \mathbf{u}_{h}+\delta \mathbf{u}_{h}\right] \delta \mathbf{u}_{h} \\
& =-\left(\boldsymbol{\psi}_{h}^{\mathrm{mv}}\right)^{T} \overline{\mathbf{R}}_{h}\left[\mathbf{u}_{h}, \mathbf{u}_{h}+\delta \mathbf{u}_{h}\right] \\
& =-\left(\boldsymbol{\psi}_{h}^{\mathrm{mv}}\right)^{T} \mathbf{R}_{h}\left(\mathbf{u}_{h}+\delta \mathbf{u}_{h}\right)+\left(\boldsymbol{\psi}_{h}^{\mathrm{mv}}\right)^{T} \mathbf{R}_{h}\left(\mathbf{u}_{h}\right) \\
& =-\left(\boldsymbol{\psi}_{h}^{\mathrm{mv}}\right)^{T} \mathbf{R}_{h}\left(\mathbf{u}_{h}+\delta \mathbf{u}_{h}\right)
\end{aligned}
$$

In the last step, $\mathbf{R}_{h}\left(\mathbf{u}_{h}\right)=0$ is used.

\section{II.D.2. Variational formulation}

In the weighted-residual formulation, the mean-value linearization can be used to define the adjoint as,

$$
\overline{\mathcal{R}}_{h}\left[\mathbf{u}_{h}, \mathbf{u}_{h}+\delta \mathbf{u}_{h}\right]\left(\mathbf{v}_{h}, \psi_{h}^{\mathrm{mv}}\right)+\overline{\mathcal{J}}_{h}\left[\mathbf{u}_{h}, \mathbf{u}_{h}+\delta \mathbf{u}_{h}\right]\left(\mathbf{v}_{h}\right)=0, \quad \forall \mathbf{v}_{h} \in \mathcal{V}_{h},
$$

where $\boldsymbol{\psi}_{h}^{\mathrm{mv}} \in \mathcal{V}_{h}$ denotes the adjoint associated with the mean-value linearization, and $\overline{\mathcal{R}}_{h}: \mathcal{V}_{h} \times$ $\mathcal{V}_{h} \rightarrow \mathbb{R}$ and $\overline{\mathcal{J}}_{h}: \mathcal{V}_{h} \rightarrow \mathbb{R}$ are defined by

$$
\begin{aligned}
\overline{\mathcal{R}}_{h}\left[\mathbf{u}_{h}, \mathbf{u}_{h}+\delta \mathbf{u}_{h}\right]\left(\mathbf{v}_{h}, \mathbf{w}_{h}\right) & =\int_{0}^{1} \mathcal{R}_{h}^{\prime}\left[\mathbf{u}_{h}+\theta \delta \mathbf{u}_{h}\right]\left(\mathbf{v}_{h}, \mathbf{w}_{h}\right) d \theta \\
\overline{\mathcal{J}}_{h}\left[\mathbf{u}_{h}, \mathbf{u}_{h}+\delta \mathbf{u}_{h}\right]\left(\mathbf{v}_{h}\right) & =\int_{0}^{1} \mathcal{J}_{h}^{\prime}\left[\mathbf{u}_{h}+\theta \delta \mathbf{u}_{h}\right]\left(\mathbf{v}_{h}\right) d \theta
\end{aligned}
$$

for $\mathbf{v}_{h}, \mathbf{w}_{h} \in \mathcal{V}_{h}$. Since

$$
\begin{aligned}
\overline{\mathcal{R}}_{h}\left[\mathbf{u}_{h}, \mathbf{u}_{h}+\delta \mathbf{u}_{h}\right]\left(\delta \mathbf{u}_{h}, \mathbf{w}_{h}\right) & =\mathcal{R}_{h}\left(\mathbf{u}_{h}+\delta \mathbf{u}_{h}, \mathbf{w}_{h}\right)-\mathcal{R}_{h}\left(\mathbf{u}_{h}, \mathbf{w}_{h}\right), \\
\overline{\mathcal{J}}_{h}\left[\mathbf{u}_{h}, \mathbf{u}_{h}+\delta \mathbf{u}_{h}\right]\left(\delta \mathbf{u}_{h}\right) & =\mathcal{J}_{h}\left(\mathbf{u}_{h}+\delta \mathbf{u}_{h}\right)-\mathcal{J}_{h}\left(\mathbf{u}_{h}\right),
\end{aligned}
$$

the output perturbation can be expressed as,

$$
\begin{aligned}
\mathcal{J}_{h}\left(\mathbf{u}_{h}+\delta \mathbf{u}_{h}\right)-\mathcal{J}_{h}\left(\mathbf{u}_{h}\right) & =\overline{\mathcal{J}}_{h}\left[\mathbf{u}_{h}, \mathbf{u}_{h}+\delta \mathbf{u}_{h}\right]\left(\delta \mathbf{u}_{h}\right) \\
& =-\overline{\mathcal{R}}_{h}\left[\mathbf{u}_{h}, \mathbf{u}_{h}+\delta \mathbf{u}_{h}\right]\left(\delta \mathbf{u}_{h}, \boldsymbol{\psi}_{h}^{\mathrm{mv}}\right) \\
& =-\mathcal{R}_{h}\left(\mathbf{u}_{h}+\delta \mathbf{u}_{h}, \boldsymbol{\psi}_{h}^{\mathrm{mv}}\right)+\mathcal{R}_{h}\left(\mathbf{u}_{h}, \boldsymbol{\psi}_{h}^{\mathrm{mv}}\right) \\
& =-\mathcal{R}_{h}\left(\mathbf{u}_{h}+\delta \mathbf{u}_{h}, \boldsymbol{\psi}_{h}^{\mathrm{mv}}\right) .
\end{aligned}
$$

In the last step, the fact that $\mathbf{u}_{h}$ is a solution to the original weighted residual statement is used, so that $\mathcal{R}_{h}\left(\mathbf{u}_{h}, \mathbf{w}_{h}\right)=0, \forall \mathbf{w}_{h} \in \mathcal{V}_{h}$. 


\section{II.E. Adjoint Implementation}

The discrete adjoint is obtained as the solution to the linear system in Eqn. 5. Since this system requires a linearization about the primal solution, the primal problem in Eqn. 1 is generally solved before the adjoint problem. Concurrent primal-adjoint solutions, however, have been investigated with certain superconvergence properties in the output estimates. ${ }^{21}$

The implementation of the adjoint solve varies depending on the structure of the code. When

the full Jacobian matrix, $\frac{\partial \mathbf{R}_{h}}{\partial \mathbf{u}_{h}}$, and an associated linear solver are available from solution of the primal problem, the transpose linear solve can be implemented in a straightforward manner. For example, if a Krylov method is used for the linear solve, the adjoint solve will require transpose applications of the matrix and the preconditioner, which generally pose little difficulty when $\frac{\partial \mathbf{R}_{h}}{\partial \mathbf{u}_{h}}$ is stored.

Conversely, when the Jacobian matrix is not stored, the discrete adjoint solve is more involved. The fact that the Jacobian matrix is transposed in Eqn. 5 means that all operations in the primal solve must be linearized, transposed, and applied in reverse order for use in the adjoint solve. For example, if a finite volume calculates residuals by reconstructing the flow state and then computing nonlinear fluxes, the adjoint residual must be obtained by first applying the transpose of the linearized fluxes and then the transpose of the reconstruction operator. Multistage solution schemes have to be modified to ensure that the adjoint solve converges and that it does so at a rate similar to that of the original linear problem. ${ }^{22}$ Implicit schemes employing point or line relaxation can also be modified to preserve discrete duality, as discussed in. ${ }^{23}$

\section{Error Estimation}

\section{III.A. Forms of Error Estimation}

The error in the solution can be quantified by various means. Discretization error is the difference between the solution to the discrete system and the exact solution to the continuous system. Its magnitude is governed by the size of the spatial and temporal mesh spacings, and it can be measured locally on individual elements or globally under a chosen norm. For general problems, the exact solution is unknown and the discretization error must be estimated, often using reconstructions based on smoothness assumptions. Another error estimate relies on the residual, which is obtained by substituting the approximate solution into the underlying partial differential equation. Nonzero residuals, calculated point-wise or integrated on an enriched space, indicate regions where the governing equations are not strongly enforced. Residual error estimates can also be expressed locally or integrated globally under a chosen norm.

For simulations of predominantly elliptic equations, such as those of structural elasticity or low-speed flows, error estimates based on local discretization errors are often sufficient. ${ }^{8}$ However, many aerospace CFD applications deal with hyperbolic equations, for which such estimates lose their efficacy. Zhang et al compare adaptive results using discretization error and residual indicators for the Euler equations. ${ }^{24}$ For one-dimensional, subsonic flows, Zhang et al find that a residual indicator is more efficient compared to a discretization-error indicator in driving the adaptation to reduce the total solution error. However, for transonic or multi-dimensional flows, neither indicator is adequately effective. In general, error estimates based on residual or discretization errors fail to capture propagation effects inherent to hyperbolic problems. ${ }^{25}$ For hyperbolic problems, the residual and discretization error may not necessarily be large in certain crucial areas that significantly affect the solution downstream. For example, for separated flow over an airfoil, small perturbations 
in certain upstream areas may have large effects on the location of the separation point, which in turn has a large effect on the calculated lift and drag. Stated another way, engineering outputs can be highly sensitive to discretization or residual errors in areas that may not be easily identifiable $a$ priori.

Fortunately, error estimates based on engineering outputs help to address these problems. Techniques for estimating errors in engineering outputs identify areas of the domain that are important for the accurate prediction of an output, accounting for propagation effects in the process. A common output error estimation technique relies on solution of the adjoint problem introduced in Section II. The resulting error estimate can be used to ascribe confidence levels to the engineering output or to drive an adaptive method with the goal of reducing the output error below a user-specified tolerance. This section reviews such existing output-error estimation techniques.

\section{III.B. The Adjoint-Weighted Residual Method}

A simplified approach to output-based error estimation begins with a solution on a "fine" discretization, $\mathbf{u}_{h} \in \mathcal{V}_{h}$, and considers the effect on the output brought about by perturbing the solution to one on a coarser discretization, $\mathbf{u}_{H} \in \mathcal{V}_{H}$. The discretization spaces are assumed to be nested, $\mathcal{V}_{H} \subset \mathcal{V}_{h}$, so that $\delta \mathbf{u}_{h}=\mathbf{u}_{H}-\mathbf{u}_{h} \in \mathcal{V}_{h}$. Such a situation is illustrated in Figure 4 for a one-dimensional finite element solution.

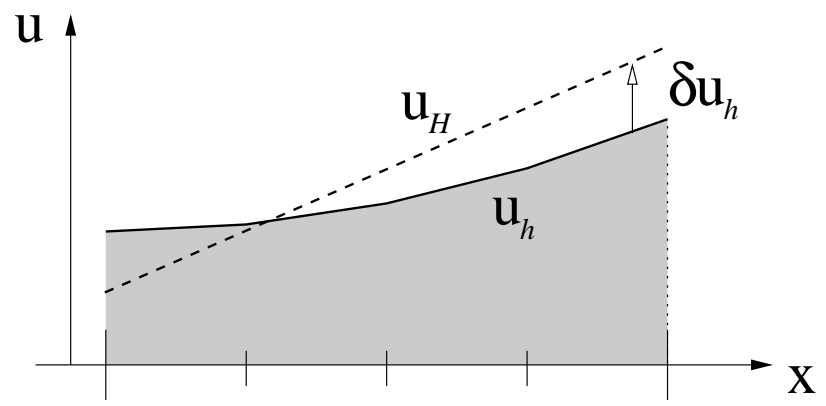

Figure 4. Comparison in one dimension of a fine solution $\mathbf{u}_{h} \in \mathcal{V}_{h}$, a coarse solution $\mathbf{u}_{H} \in \mathcal{V}_{H}$, and the difference $\delta \mathbf{u}_{h}=\mathbf{u}_{H}-\mathbf{u}_{h} \in \mathcal{V}_{h}$. In this example, the solution spaces consist of piecewise linear functions on uniform elements, and $\mathcal{V}_{h}$ is nested in $\mathcal{V}_{H}$ with four times as many elements. One coarse element is shown.

The error in $\mathcal{J}_{H}\left(\mathbf{u}_{H}\right)$ relative to $\mathcal{J}_{h}\left(\mathbf{u}_{h}\right)$ is then the output perturbation given in Eqn. 20,

$$
\begin{aligned}
\mathcal{J}_{h}\left(\mathbf{u}_{h}+\delta \mathbf{u}_{h}\right)-\mathcal{J}_{h}\left(\mathbf{u}_{h}\right) & =-\mathcal{R}_{h}\left(\mathbf{u}_{h}+\delta \mathbf{u}_{h}, \boldsymbol{\psi}_{h}^{\mathrm{mv}}\right), \\
\mathcal{J}_{H}\left(\mathbf{u}_{H}\right)-\mathcal{J}_{h}\left(\mathbf{u}_{h}\right) & =-\mathcal{R}_{h}\left(\mathbf{u}_{H}, \boldsymbol{\psi}_{h}^{\mathrm{mv}}\right),
\end{aligned}
$$

where the mean-value adjoint, $\boldsymbol{\psi}_{h}^{\mathrm{mv}}$, is used since $\delta \mathbf{u}_{h}$ is not required to be infinitesimally small. The assumption that $\mathcal{J}_{H}\left(\mathbf{u}_{H}\right)=\mathcal{J}_{h}\left(\mathbf{u}_{H}\right)$ was made above, which is generally true as long as any geometry used for the output calculation does not change between the two spaces. The name "adjoint-weighted residual method" (also referred to as the dual-weighted residual method) describes Eqn. 21: the adjoint solution weights the residual of the coarse solution to produce the error estimate.

An alternate expression for the output perturbation is obtained by defining an adjoint residual as the left hand side of Eqn. 18:

$$
\overline{\mathcal{R}}_{h}^{\psi}\left[\mathbf{u}_{h}, \mathbf{u}_{H}\right]\left(\mathbf{v}_{h}, \mathbf{w}_{h}\right) \equiv \overline{\mathcal{R}}_{h}\left[\mathbf{u}_{h}, \mathbf{u}_{H}\right]\left(\mathbf{v}_{h}, \mathbf{w}_{h}\right)+\overline{\mathcal{J}}_{h}\left[\mathbf{u}_{h}, \mathbf{u}_{H}\right]\left(\mathbf{v}_{h}\right), \quad \mathbf{v}_{h}, \mathbf{w}_{h} \in \mathcal{V}_{h}
$$


Choosing $\mathbf{v}_{h}=\delta \mathbf{u}_{h}$ and $\mathbf{w}_{h}=\mathbf{w}_{H} \in \mathcal{V}_{H}$ the adjoint residual is nonzero and is precisely the output perturbation:

$$
\begin{aligned}
\mathcal{J}_{H}\left(\mathbf{u}_{H}\right)-\mathcal{J}_{h}\left(\mathbf{u}_{h}\right) & =\mathcal{J}_{h}\left(\mathbf{u}_{h}+\delta \mathbf{u}_{h}\right)-\mathcal{J}_{h}\left(\mathbf{u}_{h}\right) \\
& =\overline{\mathcal{J}}_{h}\left[\mathbf{u}_{h}, \mathbf{u}_{H}\right]\left(\delta \mathbf{u}_{h}\right) \\
& =\overline{\mathcal{R}}_{h}^{\psi}\left[\mathbf{u}_{h}, \mathbf{u}_{H}\right]\left(\delta \mathbf{u}_{h}, \mathbf{w}_{H}\right)-\overline{\mathcal{R}}_{h}\left[\mathbf{u}_{h}, \mathbf{u}_{H}\right]\left(\delta \mathbf{u}_{h}, \mathbf{w}_{H}\right) \\
& =\overline{\mathcal{R}}_{h}^{\psi}\left[\mathbf{u}_{h}, \mathbf{u}_{H}\right]\left(\delta \mathbf{u}_{h}, \mathbf{w}_{H}\right)-\mathcal{R}_{h}\left(\mathbf{u}_{H}, \mathbf{w}_{H}\right)+\mathcal{R}_{h}\left(\mathbf{u}_{h}, \mathbf{w}_{H}\right) \\
& =\overline{\mathcal{R}}_{h}^{\psi}\left[\mathbf{u}_{h}, \mathbf{u}_{H}\right]\left(\delta \mathbf{u}_{h}, \mathbf{w}_{H}\right)
\end{aligned}
$$

where we have assumed $\mathcal{R}_{h}\left(\mathbf{u}_{H}, \mathbf{w}_{H}\right)=\mathcal{R}_{H}\left(\mathbf{u}_{H}, \mathbf{w}_{H}\right)=0 \quad \forall \mathbf{w}_{H} \in \mathcal{V}_{H}$. This assumption is satisfied for a finite element formulation using nested spaces. Note that $\mathbf{w}_{H} \in \mathcal{V}_{H}$ can be arbitrary. However, for arbitrary $\mathbf{w}_{H}$, the expression in Eqn. 23 will be highly sensitive to the global error, $\delta \mathbf{u}_{h}$, which in practice is not known exactly. This sensitivity can be reduced by using a coarse mean-value adjoint solution: $\mathbf{w}_{H}=\boldsymbol{\psi}_{H}^{\mathrm{mv}}$, where $\boldsymbol{\psi}_{H}^{\mathrm{mv}} \in \mathcal{V}_{H}$ satisfies

$$
\overline{\mathcal{R}}_{h}^{\psi}\left[\mathbf{u}_{h}, \mathbf{u}_{H}\right]\left(\mathbf{v}_{H}, \boldsymbol{\psi}_{H}^{\mathrm{mv}}\right)=0, \quad \forall \mathbf{v}_{H} \in \mathcal{V}_{H} .
$$

Subtracting Eqn. 24 from Eqn. 23 yields the output perturbation expression,

$$
\mathcal{J}_{H}\left(\mathbf{u}_{H}\right)-\mathcal{J}_{h}\left(\mathbf{u}_{h}\right)=\overline{\mathcal{R}}_{h}^{\psi}\left[\mathbf{u}_{h}, \mathbf{u}_{H}\right]\left(\delta \mathbf{u}_{h}-\mathbf{v}_{H}, \boldsymbol{\psi}_{H}^{\mathrm{mv}}\right),
$$

where $\mathbf{v}_{H} \in \mathcal{V}_{H}$ is arbitrary. The advantage of the expression in Eqn. 25 is that it is insensitive to errors in $\delta \mathbf{u}_{h}$ that lie in $\mathcal{V}_{H}$. These errors may be significant when $\mathbf{u}_{h}$ is not computed exactly, especially for hyperbolic problems, due to propagation effects. Finally, for nonlinear problems, averaging the expressions in Eqns. 21 and 25 yields an output error estimate that is more accurate by one order in the residual, compared to either expression alone. ${ }^{10,13}$

\section{III.C. Fully-Discrete Formulation}

The adjoint-weighted residual method for error estimation can also be applied in a fully-discrete formulation. Again, two discretization levels are assumed: a coarse one with $N_{H}$ degrees of freedom, and a fine one with $N_{h}$ degrees of freedom. A representation of $\mathbf{u}_{H}$ on the fine space is assumed to be given by $\mathbf{u}_{h}^{H} \equiv \mathbf{I}_{h}^{H} \mathbf{u}_{H}$, where $\mathbf{I}_{h}^{H} \in \mathbb{R}^{N_{h} \times N_{H}}$ is a suitably-chosen prolongation matrix. In a standard finite element discretization, $\mathbf{I}_{h}^{H}$ is the natural injection operator. In the general case, $\mathbf{I}_{h}^{H}$ should be consistent with the discretization used.

Setting $\delta \mathbf{u}_{h}=\mathbf{I}_{h}^{H} \mathbf{u}_{H}-\mathbf{u}_{h}$, we can express the output perturbation seen on the fine level using Eqn. 17

$$
J_{H}\left(\mathbf{u}_{H}\right)-J_{h}\left(\mathbf{u}_{h}\right)=-\left(\boldsymbol{\psi}_{h}^{\mathrm{mv}}\right)^{T} \mathbf{R}_{h}\left(\mathbf{u}_{h}^{H}\right),
$$

where $J_{H}\left(\mathbf{u}_{H}\right)=J_{h}\left(\mathbf{u}_{h}^{H}\right)$ is assumed.

Alternately, defining the discrete adjoint residual $\overline{\mathbf{R}}_{h}^{\psi}\left(\mathbf{w}_{h}\right) \in \mathbb{R}^{N_{h}}, \mathbf{w}_{h} \in \mathbb{R}^{N_{h}}$ as

$$
\overline{\mathbf{R}}_{h}^{\psi}\left(\mathbf{w}_{h}\right) \equiv\left(\overline{\mathbf{R}}_{h}\left[\mathbf{u}_{h}, \mathbf{u}_{h}^{H}\right]\right)^{T} \mathbf{w}_{h}+\left(\bar{J}_{h}\left[\mathbf{u}_{h}, \mathbf{u}_{h}^{H}\right]\right)^{T},
$$

leads to an error estimate based on the adjoint residual,

$$
\begin{aligned}
J_{H}\left(\mathbf{u}_{H}\right)-J_{h}\left(\mathbf{u}_{h}\right) & =\bar{J}_{h}\left[\mathbf{u}_{h}, \mathbf{u}_{h}^{H}\right] \delta \mathbf{u}_{h} \\
& =\left(\overline{\mathbf{R}}_{h}^{\psi}\left(\mathbf{w}_{h}\right)\right)^{T} \delta \mathbf{u}_{h}-\mathbf{w}_{h}^{T} \overline{\mathbf{R}}_{h}\left[\mathbf{u}_{h}, \mathbf{u}_{h}^{H}\right] \delta \mathbf{u}_{h} \\
& =\left(\overline{\mathbf{R}}_{h}^{\psi}\left(\mathbf{w}_{h}\right)\right)^{T} \delta \mathbf{u}_{h}-\mathbf{w}_{h}^{T} \mathbf{R}_{h}\left(\mathbf{u}_{h}^{H}\right) .
\end{aligned}
$$


This equation is satisfied for arbitrary $\mathbf{w}_{h} \in \mathbb{R}^{N_{h}}$. Similarly to the variational formulation, reduced sensitivity to errors in $\delta \mathbf{u}_{h}$ is attained by setting $\mathbf{w}_{h}=\mathbf{I}_{h}^{H} \boldsymbol{\psi}_{H}^{\mathrm{mv}}$, where the coarse mean-value adjoint solution is defined similarly to Eqn. 24. Again, for nonlinear problems, averaging the expressions in Eqns. 21 and 25 yields an output error estimate that is more accurate compared to either expression alone.

\section{III.D. Approximating $\psi_{h}^{\mathrm{mv}}, \mathbf{u}_{h}$}

Evaluating the output perturbation in Eqn. 21 requires a residual evaluation on the fine space $\mathcal{V}_{h}$, weighted by the mean-value adjoint, $\boldsymbol{\psi}_{h}^{\mathrm{mv}}$. A residual evaluation on $\mathcal{V}_{h}$ is tractable, but solving Eqn. 15 to calculate $\boldsymbol{\psi}_{h}^{\mathrm{mv}}$ requires both a primal and an adjoint solve on $\mathcal{V}_{h}$. Similarly, the expression in Eqn. 25 requires the primal solution $\mathbf{u}_{h}$. These calculations on $\mathcal{V}_{h}$ are expensive and defeat the purpose of estimating the error since $\mathcal{J}_{H}-\mathcal{J}_{h}$ could be calculated directly if $\mathbf{u}_{h}$ were available. However, such an approach can still be useful for obtaining an accurate indicator for adaptation and an improved final solution. ${ }^{26}$

In practice, exact solutions on $\mathcal{V}_{h}$ are treated as computationally intractable and hence $\mathbf{u}_{h}$ and $\boldsymbol{\psi}_{h}^{\mathrm{mv}}$ must be approximated. Readily available through calculations on $\mathcal{V}_{H}$ are $\mathbf{u}_{H}$ and $\boldsymbol{\psi}_{H} \in \mathcal{V}_{H}$, where

$$
\mathcal{R}_{H}^{\prime}\left[\mathbf{u}_{H}\right]\left(\mathbf{v}_{H}, \boldsymbol{\psi}_{H}\right)+\mathcal{J}_{H}^{\prime}\left[\mathbf{u}_{H}\right]\left(\mathbf{v}_{H}\right)=0, \quad \forall \mathbf{v}_{H} \in \mathcal{V}_{H} .
$$

One approach for approximating $\mathbf{u}_{h}$ is to reconstruct $\mathbf{u}_{H}$ on $\mathcal{V}_{h}$ using a higher-accuracy stencil. In the finite element setting, this could be least squares patch reconstruction. ${ }^{5,13,20,27}$ Reconstruction is based on a smoothness assumption and loses validity near discontinuities. In addition, without limiting, no guarantees exist that reconstructed solutions will remain physical for nonlinear problems. An alternate approach is therefore to project $\mathbf{u}_{H}$ into $\mathcal{V}_{h}$ and to apply several steps of an iterative solution scheme. ${ }^{16,28}$ In either case, the difference between the approximated $\mathbf{u}_{h}$ and $\mathbf{u}_{H}$ can be used directly in Eqn. 25 to compute the error estimate.

$\boldsymbol{\psi}_{h}^{\mathrm{mv}}$ can be approximated in several ways. Just as $\mathbf{u}_{h}$, it can be reconstructed from $\boldsymbol{\psi}_{H}$ using a higher-accuracy stencil. ${ }^{13,20,27,29}$ Alternately, it can be the result of an exact adjoint solve ${ }^{19}$ or several iterative solution steps ${ }^{16,28}$ on $\mathcal{V}_{h}$. The linearization for the adjoint problem can be performed: (1) about the projected $\mathbf{u}_{H} ;(2)$ about the approximated $\mathbf{u}_{h} ;$ (3) about a sequence of states between $\mathbf{u}_{H}$ and $\mathbf{u}_{h}$ chosen to approximate the mean-value integrals in Eqn. 19 numerically. ${ }^{20}$ Numerical experiments do not indicate substantial differences between these approaches. Finally, note that errors made in approximating $\boldsymbol{\psi}_{h}^{\mathrm{mv}}$ using the above approaches are not guaranteed to be negligible in the vicinity of under-resolved flow features. Care must be taken in working with error estimates obtained in these cases.

\section{III.E. Computable Corrections and Remaining Error}

In the variational formulation, if $\boldsymbol{\psi}_{h}^{\mathrm{mv}} \in \mathcal{V}_{h}$ is decomposed as $\boldsymbol{\psi}_{h}^{\mathrm{mv}}=\boldsymbol{\psi}_{H}^{\mathrm{mv}}+\delta \boldsymbol{\psi}_{h}^{\mathrm{mv}}$, where $\boldsymbol{\psi}_{H}^{\mathrm{mv}} \in \mathcal{V}_{H}$, then by Galerkin orthogonality Eqn. 21 becomes

$$
\mathcal{J}_{H}\left(\mathbf{u}_{H}\right)-\mathcal{J}_{h}\left(\mathbf{u}_{h}\right)=-\mathcal{R}_{h}\left(\mathbf{u}_{H}, \boldsymbol{\psi}_{H}^{\mathrm{mv}}+\delta \boldsymbol{\psi}_{h}^{\mathrm{mv}}\right)=-\mathcal{R}_{h}\left(\mathbf{u}_{H}, \delta \boldsymbol{\psi}_{h}^{\mathrm{mv}}\right),
$$

The discrete analog, if we do not assume Galerkin orthogonality, is

$$
J_{H}\left(\mathbf{u}_{H}\right)-J_{h}\left(\mathbf{u}_{h}\right)=\underbrace{-\left(\mathbf{I}_{h}^{H} \boldsymbol{\psi}_{H}^{\mathrm{mv}}\right)^{T} \mathbf{R}_{h}\left(\mathbf{I}_{h}^{H} \mathbf{u}_{H}\right)}_{\text {computable correction }} \underbrace{-\left(\delta \boldsymbol{\psi}_{h}^{\mathrm{mv}}\right)^{T} \mathbf{R}_{h}\left(\mathbf{I}_{h}^{H} \mathbf{u}_{h}^{H}\right)}_{\text {remaining error }}
$$


where $\boldsymbol{\psi}_{h}^{\mathrm{mv}}=\mathbf{I}_{h}^{H} \boldsymbol{\psi}_{H}^{\mathrm{mv}}+\delta \boldsymbol{\psi}_{h}^{\mathrm{mv}}$. The first term, which would be zero if the discretization arose from a variational formulation, is often called the computable correction. In particular, it is nonzero for reconstruction-based finite volume schemes. Note that Eqn. 29 yields the same result as Eqn. 26 - the only difference is in the bookkeeping. If a code is written to incorporate the computable correction into its outputs, the remaining error effectively becomes the error estimate.

\section{III.F. Error Effectivity}

In the limit of a very fine (and consistent) discretization, " $h \rightarrow 0$ " and $\mathbf{u}_{h} \rightarrow \mathbf{u}$, Eqns. 21 and 25 yield the true output error in the solution: $\mathcal{J}_{H}\left(\mathbf{u}_{H}\right)-\mathcal{J}(\mathbf{u})$. In practice, however, a finite dimensional $\mathcal{V}_{h}$ is employed, obtained from $\mathcal{V}_{H}$ by uniform refinement or interpolation order increase. Hence, the calculated output error is generally not equal to and not a bound for the true error. It is an estimate whose accuracy depends on the enrichment of $\mathcal{V}_{h}$ relative to $\mathcal{V}_{H}$. Indeed, the choice of enrichment governs the behavior of the error effectivity,

$$
\eta_{H}^{e} \equiv \frac{\mathcal{J}_{H}\left(\mathbf{u}_{H}\right)-\mathcal{J}_{h}\left(\mathbf{u}_{h}\right)}{\mathcal{J}_{H}\left(\mathbf{u}_{H}\right)-\mathcal{J}(\mathbf{u})}
$$

An effectivity close to 1 is desirable. However, not all enrichment strategies will guarantee this asymptotically as $H \rightarrow 0$. For example, if $H$ denotes mesh size and the output error converges as $\mathcal{J}_{H}\left(\mathbf{u}_{H}\right)-\mathcal{J}(\mathbf{u})=C H^{k}$, a choice of $h=H / 2$ for the enriched space yields an asymptotic effectivity of $\eta_{H}^{e}=1-(1 / 2)^{k}$. A similar result holds for order enrichment on a fixed mesh. However if $H$ denotes mesh size but $\mathcal{V}_{h}$ is obtained from $\mathcal{V}_{H}$ by order enrichment of $\delta p$, the effectivity behaves as $\eta_{H}^{e}=1-C H^{\delta p} \rightarrow 1$ as $H \rightarrow 0$

\section{III.G. Impact of Shocks}

Shock waves (or other under-resolved phenomena) can present a variety of problems when estimating errors. For example, estimation of $\mathbf{u}_{h}$ through reconstruction can introduce oscillations that contaminate error estimates. This contamination can be reduced by using monotonic reconstruction procedures.

Another issue is the use of shock-capturing stabilization terms in the discretization that are non-zero even when acting upon the exact solution. In these situations, the semilinear form is inconsistent since $\mathcal{R}_{h}(\mathbf{u}, \mathbf{v}) \neq 0$ for all $\mathbf{v} \in \mathcal{V}$. However, for the method to be convergent, the stabilization terms are assumed to asymptote to zero as $h \rightarrow 0$. In other words, the method has asymptotic primal consistency.

The error due to asymptotically consistent stabilization terms can be estimated by separating the weighted residual statement into consistent and asymptotically consistent parts,

$$
\mathcal{R}_{h}\left(\mathbf{u}_{h}, \mathbf{v}_{h}\right)+\mathcal{R}_{h}^{\epsilon}\left(\mathbf{u}_{h}, \mathbf{v}_{h}\right)=0, \quad \forall \mathbf{v}_{h} \in \mathcal{V}_{h}
$$

where $\mathcal{R}_{h}(\cdot, \cdot)$ is a consistent semilinear form, and $\mathcal{R}_{h}^{\epsilon}(\cdot, \cdot)$ is an asymptotically consistent form. Then, using Eqn. 8, the output error due to using asymptotic consistent stabilization is

$$
\delta \mathcal{J}_{h}^{\epsilon}=\mathcal{R}_{h}^{\epsilon}\left(\mathbf{u}_{h}, \boldsymbol{\psi}_{h}\right)
$$

where $\psi_{h}$ is the solution to Eqn. 10 when the residual perturbation is approximated as infinitesimally small. When performing error estimation, approximations to $\mathbf{u}_{h}$ and $\boldsymbol{\psi}_{h}$ are available, and hence $\delta \mathcal{J}_{h}^{\epsilon}$ is computable. 


\section{Mesh Adaptation}

A typical adaptive solution process is illustrated in Figure 5. The input is an initial coarse mesh

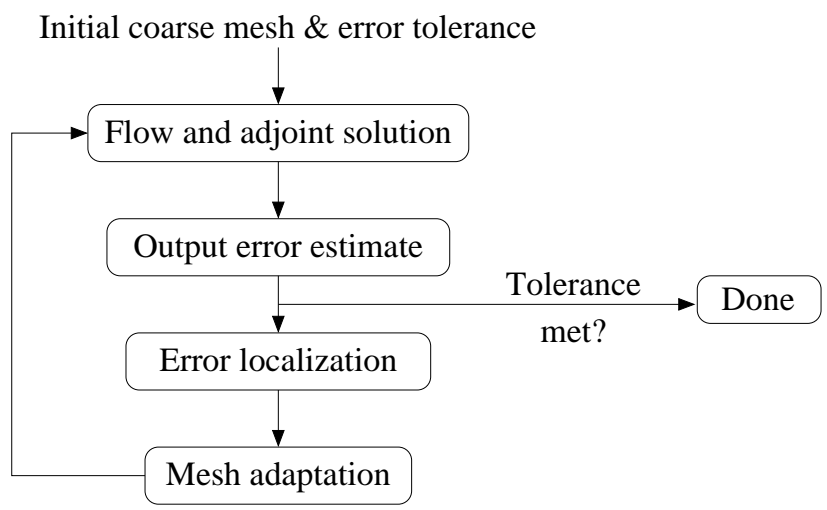

Figure 5. Adaptive solution process flowchart. The input consists of an initial coarse mesh and a requested error tolerance. Adaptation stops when the error tolerance is met.

along with a user-prescribed error tolerance for an output. The iterative process starts by solving the primal and adjoint problems on the initial coarse mesh. Next, the output error is estimated using the adjoint-weighted residual method described in Section III.B. If the global error tolerance criterion is met, the adaptive process terminates. Otherwise, the error estimate is localized to the elements, and the mesh is adapted. The process then repeats until the tolerance is met.

In output-based error estimation, the error localization is fairly straightforward. However, numerous strategies exist for translating the error indicator into a modified mesh. In CFD, the most popular adaptation strategy is $h$-adaptation, in which only the triangulation forming the mesh is varied. In high-order methods, additional strategies include $p$-adaptation, in which the interpolation order is changed on a fixed triangulation, ${ }^{13,30}$ and $h p$-adaptation in which both the order and the triangulation are varied. ${ }^{31-35}$ For CFD applications, in which solutions often possess localized, singular features, $h$-adaptation is key to an efficient adaptation strategy. In addition, most practical codes operate at one or a limited number of orders, making $h$-adaptation the only practical approach. With the growing popularity of high-order methods, however, $h p$-adaptation will be an important strategy for increased efficiency in the future. This section reviews general aspects of $h$-adaptation, and presents specific applications to output-based adaptation.

\section{IV.A. Error Localization}

The output error estimates in Eqns. 21 and 26 consist of a residual evaluation on the refined space $\mathcal{V}_{h}$, using $\boldsymbol{\psi}_{h}^{\mathrm{mv}}$ as the test function. In a finite element or finite volume method, this residual evaluation is a sum over all elements/volumes in the fine space. Since the coarse and fine spaces are assumed nested, the error estimate can be written as,

$$
\mathcal{J}_{H}\left(\mathbf{u}_{H}\right)-\mathcal{J}_{h}\left(\mathbf{u}_{h}\right)=-\sum_{\kappa_{H} \in T_{H}} \sum_{\kappa_{h} \in \kappa_{H}} \mathcal{R}_{h}\left(\mathbf{u}_{H},\left.\boldsymbol{\psi}_{h}^{\mathrm{mv}}\right|_{\kappa_{h}}\right)
$$

where $T_{H}$ is the coarse triangulation, $\kappa_{H} / \kappa_{h}$ is an element of the coarse/fine triangulation, and $\left.\right|_{\kappa_{h}}$ refers to restriction to element $\kappa_{h}$. The discrete version is analogous. Note that the coarse/fine spaces can consist of the same triangulation, for example when only the interpolation order is 
increased, in which case $\kappa_{H}=\kappa_{h}$. Eqn. 33 expresses the output error in terms of contributions from each coarse element. A common approach for obtaining an error indicator is to take the absolute value of the elemental contribution, ${ }^{10,12,19,20,29}$

$$
\epsilon_{\kappa_{H}}=\left|\sum_{\kappa_{h} \in \kappa_{H}} \mathcal{R}_{h}\left(\mathbf{u}_{H},\left.\boldsymbol{\psi}_{h}^{\mathrm{mv}}\right|_{\kappa_{h}}\right)\right| .
$$

In some works, this error indicator is averaged with a similar estimate derived from the adjoint equation residual, Eqn. 25, ${ }^{29,36,37}$

$$
\epsilon_{\kappa_{H}}^{\psi}=\left|\sum_{\kappa_{h} \in \kappa_{H}} \overline{\mathcal{R}}_{h}^{\psi}\left[\mathbf{u}_{h}, \mathbf{u}_{H}\right]\left(\left.\delta \mathbf{u}_{h}\right|_{\kappa_{h}}, \psi_{H}\right)\right| .
$$

This indicator targets areas of nonzero adjoint residual, weighted by a primal interpolation error estimate. Note, $\boldsymbol{\psi}_{H}^{\mathrm{mv}}$ from Eqn. 25 has been approximated by the readily-computable coarse adjoint solution $\boldsymbol{\psi}_{H}$ from Eqn. 28. Numerical experiments have shown that the two error indicators, $\epsilon_{\kappa_{H}}$ and $\epsilon_{\kappa_{H}}^{\psi}$, yield similar mesh distributions when used to drive adaptation.

The above error localization is applicable to finite volume and discontinuous Galerkin discretizations, since residuals vanish locally on each element for these discretizations. Thus, no systematic inter-element error cancellation is expected in the output error estimate, Eqn. 33, and the absolute value signs in Eqns. 34 and 35 are justified. However, local residuals do not necessarily vanish for continuous finite element discretizations. Consider for example a continuous finite element discretization of Poisson's equation, in which the elemental contributions to the residual contain terms of the form $\int_{\kappa_{h}} \nabla \mathbf{u}_{H} \cdot \nabla \boldsymbol{\psi}_{h}^{\mathrm{mv}}$. Simply placing absolute value signs around these terms to obtain the elemental error indicator would lead to a systematic over-estimate of the output error via a sum of the indicators. This over-estimate is due to a poor bookkeeping choice for the error and can be fixed by integrating the residual terms by parts on each element. The result is a set of element-interior terms containing the strong form of the residual, and a set of face flux jump terms, which are present because the gradient of $\mathbf{u}_{H}$ is not continuous. Both of these terms are expected to go to zero with mesh refinement, and the flux jump terms will dominate for low orders. ${ }^{38}$ The face flux residuals can be pushed back onto the elements by assigning half of the flux residual to each of the two elements adjacent to the face. ${ }^{27}$ With the output error split into element and face contributions, each with absolute value signs, the elemental error indicator is now sensitive to variations in $\boldsymbol{\psi}_{H}^{\mathrm{mv}}$ in the coarse space $\mathcal{V}_{H}$. To minimize this variation, the adjoint error, $\boldsymbol{\psi}_{H}^{\mathrm{mv}}-\boldsymbol{\psi}_{H}$, is used instead of $\boldsymbol{\psi}_{H}^{\mathrm{mv}}$ alone to weight the primal residual. ${ }^{10}$ Note that by Galerkin orthogonality, this change has no effect on the output error estimate in Eqn. 33. Finally, for convection equations, the continuity of $\mathbf{u}_{H}$ eliminates the need for interior flux residuals, although inflow flux residuals are still required and the stabilization terms must be treated appropriately. ${ }^{39}$

For systems of equations, indicators are typically computed separately for each equation and summed together. Due to the absolute values, the sum of the error indicators, $\epsilon=\sum_{\kappa_{H}} \epsilon_{\kappa_{H}}$, is greater or equal to the original output error estimate. However, it is not a bound on the actual error, or even on $J_{H}\left(\mathbf{u}_{H}\right)-J_{h}\left(\mathbf{u}_{h}\right)$, because of the approximations made in the derivation. In practice, the validity of the approximations improves with refinement, and the above estimate becomes an accurate measure of the true error.

\section{IV.B. $h$-Adaptation Mechanics}

Many approaches to adapt a mesh rely upon the application of local mesh operators through which the mesh is modified incrementally. A simple example of a local operator is element sub- 
division in a setting that supports non-conforming, or hanging, nodes. ${ }^{27,34,40-42}$ For triangular and tetrahedral meshes, local mesh modification operators consist of node insertion, face/edge swapping, edge collapsing, and node movement. These operators have been studied extensively by various authors ${ }^{43-49}$ in different contexts. The primary advantage of local operators is their robustness: the entire mesh is not regenerated all at once, but rather each operator affects only a prescribed number of nodes, edges, or elements.

Another approach to adapt a mesh is global re-meshing, in which a new mesh is generated for the entire computational domain. The original, or background, mesh is used to store desired mesh characteristics during re-generation. For applications to adaptation, the desired mesh characteristics are often described using a Riemannian metric, the idea being that in an optimal mesh, all edge lengths will have unit measure under the metric. ${ }^{44,45}$ In a Cartesian coordinate system, an infinitesimal segment $\delta \mathbf{x}$ has length $\delta \Gamma$ under a Riemannian metric $\mathbf{M}$,

$$
\delta \Gamma^{2}=\delta \mathbf{x}^{T} \mathbf{M} \delta \mathbf{x}=\delta x_{i} M_{i j} \delta x_{j},
$$

where $\delta x_{i}$ are the components of $\delta \mathbf{x} \in \mathbb{R}^{d}$ and $M_{i j}$ are the components of the symmetric, positive definite $\mathbf{M} \in \mathbb{R}^{d \times d}$.

The metric $\mathbf{M}$ contains information on the desired mesh edge lengths in physical space. As $\mathbf{M}$ is symmetric and positive definite, the unit measure requirement,

$$
\mathbf{x}^{T} \mathbf{M} \mathbf{x}=1
$$

describes an ellipsoid in physical space that maps to a sphere under the action of the metric. The eigenvectors of $\mathbf{M}$ form the orthogonal axes of the ellipsoid - i.e. the principal directions. The corresponding eigenvalues, $\lambda_{i}$, are related to the lengths of the axes, $h_{i}$, via

$$
\lambda_{i}=\frac{1}{h_{i}^{2}} \Rightarrow \frac{h_{i}}{h_{j}}=\left(\frac{\lambda_{j}}{\lambda_{i}}\right)^{1 / 2}
$$

Physically, the $h_{i}$ are the principal stretching magnitudes. A diagram of a possible ellipse resulting from the unit-measure requirement in two dimensions is given in Figure 6. Thus, the ratio of eigenvalues of $\mathbf{M}$ can be used to define a desired level of anisotropy.

A successful approach for generating simplex meshes based on a Riemannian metric is mapped Delaunay triangulation, in which a Delaunay mesh generation algorithm ${ }^{50}$ is applied in the mapped space, allowing for the creation of stretched and variable size triangles or tetrahedra. ${ }^{51}$ This method is implemented in the Bi-dimensional Anisotropic Mesh Generator (BAMG), ${ }^{52,53}$ which has been used in various finite volume $\mathrm{e}^{54,55}$ and discontinuous Galerkin ${ }^{16,28,36}$ applications requiring anisotropic meshes. Examples of output-adapted meshes obtained using BAMG are shown in Figures 10, 20, and 22.

\section{IV.C. Overview of Adaptation Strategies}

In $h$-adaptation, the determination of which elements to refine or coarsen has important implications for practical simulations: too little refinement at each adaptation iteration may result in an unnecessary number of iterations; too much refinement may ask for an expensive solve on an overly-refined mesh.

Aftosmis and Berger discuss adaptation strategies in terms of error distribution histograms, ${ }^{56}$ in which elements are binned according to the error indicator (Eqn. 34 for output-based adaptation). 


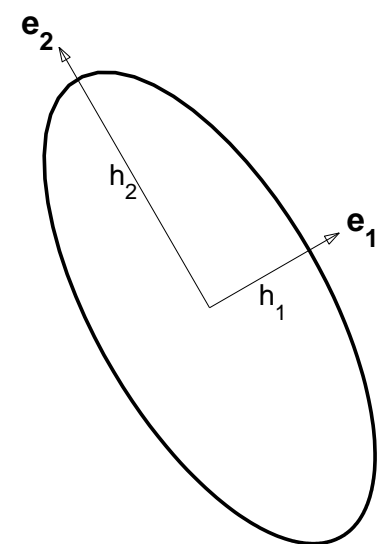

Figure 6. Ellipse representing requested mesh sizes implied by equal measure under a Riemannian metric M. Also shown are the principal directions, $\mathbf{e}_{i}$, and the associated principal stretching magnitudes, $h_{i}$.

The assumption made in virtually all adaptation strategies is that in an ideal mesh the userprescribed error tolerance is satisfied and the error is equidistributed among the elements. ${ }^{47}$ This situation corresponds to a "delta" histogram, in which all elements lie in the same bin. In contrast, the initial coarse mesh will generally have some distribution of error indicators, as illustrated in Figure 7 . The goal of an adaptation strategy is then to drive the histogram towards the ideal delta
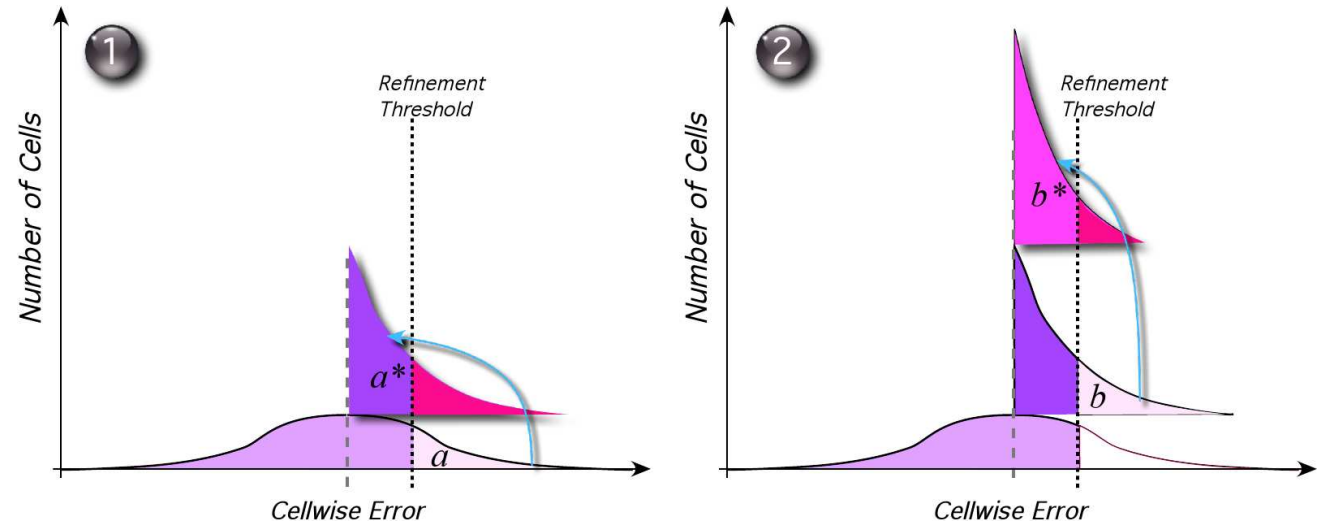

Figure 7. Sample error indicator histogram and a constant-threshold refinement strategy. Reproduced with permission from. ${ }^{57}$

distribution using a prescribed adaptation strategy.

Nemec et $a l^{57}$ discuss two adaptation strategies based on either a constant or a decreasing refinement threshold. In a constant threshold strategy, depicted in Figure 7, all elements with error above a certain fixed value are refined. This strategy is simple but potentially expensive: initial refinement targets virtually all of the elements and leads to a rapid growth in the mesh size in the first few iterations, while elements with the highest error (in the right tail of the histogram) are likely to be among the last elements to have their error reduced to the target level. In contrast, with a decreasing threshold, shown in Figure 8, elements with the highest error are targeted for 
refinement first, so that the mesh size grows more slowly and multiple expensive solves on the finest meshes are avoided.
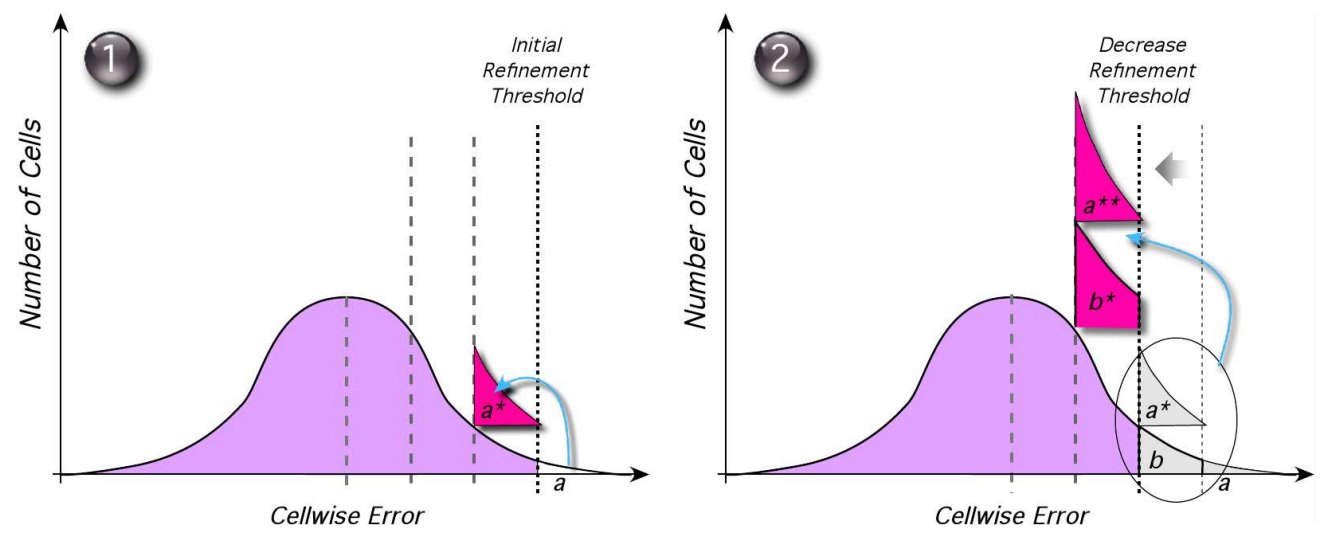

Figure 8. Adaptation strategy using a decreasing threshold. Reproduced with permission from. ${ }^{57}$

Most adaptation optimization methods follow some variation of a decreasing threshold strategy. For example, a fixed-fraction approach prescribes a fraction of elements with the highest error indicator to be refined at each adaptation iteration, such that the decreasing threshold is a function of the shape of the error histogram. Then, the elements targeted for adaptation are typically refined in a locally uniform manner, e.g. by splitting all edges in half. This simple approach has been applied to output-based adaptation in several studies ${ }^{19,20,26,35,58,59}$ The fixed-fraction parameter is often chosen heuristically in a trade-off between an excessive number of iterations and a risk of over-refinement. Nevertheless, the method works quite well for practical problems.

\section{IV.D. Incorporating a priori Analysis and Anisotropy}

The fixed-fraction adaptive strategy with locally uniform refinement does not account for the rate at which the error decreases with mesh refinement in a given adaptive iteration. This disregard for the error convergence rate could lead to over-refinement of the mesh or to an excessive number of adaptive iterations to achieve the desired target error. Adaptation strategies have been developed that attempt to meet the global tolerance while equidistributing the error among elements through the incorporation of a priori error analysis. In the context of isotropic, output-based adaptation, Venditti and Darmofal ${ }^{29}$ developed such a method based on the previous work of Zienkiewicz and Zhu. ${ }^{60}$ In this method a permissible element error $e_{\kappa}=e_{0} / N$ is defined at each adaptation iteration, where $e_{0}$ is the user tolerance, and $N$ is the current number of elements. Coupled with an a priori error estimate that the error converges as $\mathcal{O}\left(h^{r}\right)$, where $r$ is the a priori estimated convergence rate, element size requests can be made that equidistribute the error.

An important ingredient in $h$-adaptation for aerodynamic computations is the ability to generate stretched elements in areas such as boundary layers, wakes, and shocks where the solution exhibits anisotropy, which refers to variations of disparate magnitudes in different directions. The first output-based adaptive method to incorporate anisotropy was proposed by Venditti and Darmofal $^{55,61}$ and applied to a nominally second-order accurate finite volume algorithm. Their approach was to combine the isotropic, output-based approach using a priori estimates with existing solution Hessian-based methods for anisotropic adaptation. 
For spatially second-order methods, the dominant method for detecting anisotropy involves estimating the Hessian matrix, $\mathbf{H}$, of a scalar solution, $u .^{44,45,51,62}$ The components of $\mathbf{H}$ are given by

$$
H_{i j}=\frac{\partial^{2} u}{\partial x_{i} \partial x_{j}}, \quad i, j \in[1, . ., d], \quad d=\text { dimension }
$$

The second derivatives can be estimated by, for example, a quadratic reconstruction of a linear solution. For the Euler or Navier-Stokes equations, the Hessian of the Mach number has been found to perform sufficiently well as the scalar $u$.

The metric is obtained from the Hessian by requiring that the interpolation error estimate of the scalar quantity $u$ be the same in any chosen spatial direction. For linear interpolation of $u$ along the segment $\delta \mathbf{x}$, the maximum interpolation error can be bounded by the second derivative of $u$ along $\delta \mathbf{x}$. The Hessian matrix stores precisely this information, so that requiring the interpolation error bound to be approximately constant independent of the direction of $\delta \mathbf{x}$, leads to the metric choice

$$
\mathbf{M}=C|\mathbf{H}|,
$$

where $C$ is a constant independent of direction, and $|\mathbf{H}|$ is the positive, semi-definite form of the Hessian: $|\mathbf{H}|=\mathbf{V}|\boldsymbol{\Lambda}| \mathbf{V}^{-1}$ for $\mathbf{H}=\mathbf{V} \boldsymbol{\Lambda} \mathbf{V}^{-1}$. Two intervals, $\delta \mathbf{x}_{1}$ and $\delta \mathbf{x}_{2}$, having the same measure under this $\mathbf{M}$ will have the same estimated interpolation error bounds.

To fully define the metric, the absolute mesh size, i.e. the constant $C$ in Eqn. 37, has to be fixed. While in pure Hessian-based adaptation a global value for $C$ is used, the output-based method of Venditti and Darmofal sets $C$ locally according to the output error indicator. ${ }^{55}$ As a result, the smallest mesh length is controlled by the output error indicator while the anisotropy is controlled by the solution Hessian.

The definition of a metric tensor becomes difficult for high-order methods because the standard Hessian matrix approach assumes linear interpolation of the scalar quantity. For general order $p$ interpolation, the interpolation error is governed by the order $p+1$ derivatives. Thus, one possible extension of the Hessian approach is based on constructing a metric around the direction of maximum $p+1$ st derivative. ${ }^{5,63}$

An additional problem with higher-order discretization is that the need for curved mesh elements and high-fidelity geometry representations. Recent work by Oliver ${ }^{16}$ explores a novel implementation approach for high-order metric-driven meshing, in which the adaptation is performed on a mapped linear-triangle mesh. An elasticity analogy is then used to transform the linear mesh to a curved, boundary-conforming mesh around the true geometry. The robustness of this approach relies on the success of the linear meshing, which may not be guaranteed for highly-anisotropic boundary-layer meshes.

The metric tensor may also be used to guide an adaptation procedure based on local operators. In the context of pure Hessian-based adaptation, Diaz et al ${ }^{44}$ present a two-dimensional algorithm that uses the metric-based edge length to decide which operation to apply. Specifically, edge splitting, edge collapsing, edge swapping, and node movement are applied to make all edges approximately the same length when measured using the metric tensor. Habashi et $a l^{45}$ and Xia et $a l^{46}$ present similar algorithms, with slight modifications in Hessian definition and in the local operators. Park ${ }^{48,49}$ extends these local mesh modification operators to output-based mesh adaptation, in both two and three dimensions. 


\section{IV.E. Direct Optimization Adaptation}

The output-based adaptive approaches described in Section IV.D rely upon a priori analysis to estimate desired grid characteristics. Further, the interpolation error assumptions are made without regard to the output of interest by using a single scalar, such as the Mach number, to control the anisotropy for a system of equations. While the Mach number choice has worked well so far, it is arbitrary. Diaz et $a l^{44}$ propose choosing an intersection of metrics derived from all variables in the system, although this choice relies on the variables used (e.g. conservative versus primitive), and using more variables can make the resulting intersected metric too restrictive. More generally, for output-based adaptation, the assumption that the directional interpolation error must be equidistributed for one or more scalar variables at each point in the domain may not be valid. Of interest are only the interpolation errors that create residuals that affect the output. This observation has motivated research into adaptation algorithms that more directly target the error indicator.

Formaggia et $a l^{64}$ combine Hessian-based interpolation error estimates with output-based $a$ posteriori error analysis to arrive at an output-based error indicator that explicitly includes the anisotropy of each element. However, for the purpose of mesh adaptation, a metric is still defined using the resulting element modification requests. Schneider and Jimack ${ }^{65}$ calculate the sensitivities of the output error estimate with respect to node positions and formulate an optimization problem to reduce the output error estimate by redistributing the nodes. The sensitivities with respect to node positions are calculated efficiently by solving an additional adjoint problem. This approach directly targets the output error estimate and automatically leads to anisotropic meshes where appropriate. Schneider and Jimack then combine this node repositioning with isotropic local mesh refinement sequentially in a hybrid optimization/adaptation algorithm.

Park $^{37}$ introduces an algorithm that directly targets the output error through local mesh operators of element swapping, node movement, element collapse, and element splitting. Using the output error indicator to rank elements and nodes, these operations are performed in sequence and automatically result in mesh anisotropy. The details of the adaptation are also given in an earlier work, in the context of interpolation error. ${ }^{66}$ While the grids produced by this technique lack the regularity of those produced using metric-based adaptation, their accuracy is comparable.

\section{IV.F. Cut-Cell Methods}

A successful adaptation algorithm relies on automation and robustness of the mesh generation or modification. Standard boundary-conforming meshers must ensure both geometry fidelity and mesh validity, a task that becomes difficult, for example, for anisotropic meshes around curved geometries. An alternate approach to mesh generation is the class of cut-cell methods, in which the computational domain is formed by intersecting the geometry of interest with a volume-filling background mesh. Without the boundary-conforming constraint, generation or adaptation of the volume-filling background mesh is straightforward. However, the burden of robustness is transferred to the computational geometry problem of intersecting the background mesh with the geometry.

The most common cut-cell technique is the Cartesian method, a name that refers to the rectangular or hexahedral cells on a regular lattice used for the background mesh (see Figure 9). The Cartesian method was pioneered in the early days of $\mathrm{CFD},{ }^{67-71}$ has been used in industry, ${ }^{72-74}$

and is the subject of ongoing research. ${ }^{59}$ Recently, the cut-cell technique has also been applied to simplex background meshes, which provide greater flexibility in directional resolution. ${ }^{63,66,75}$

The advantage of cut-cell methods for mesh adaptation is the automation that results from 


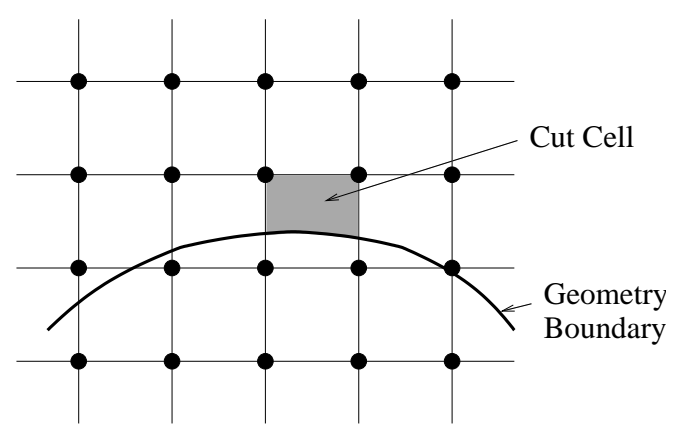

Figure 9. Sample Cartesian mesh in two dimensions. The square lattice mesh does not conform to the geometry. Cut cells are portions of intersected elements that lie inside the computational domain (above the geometry boundary in this case).

removing the boundary-conforming constraint. In a Cartesian method, hanging-node refinement is the single practical option for adaptation, and has been implemented efficiently. ${ }^{76}$ In simplex cut-cell methods, adaptation can also be performed through global re-meshing ${ }^{63}$ or through local operators. ${ }^{66}$ Cut-cell methods have been successfully applied to output-based adaptive simulations of Euler flows and to moderate Reynolds number viscous flows. For boundary-layer viscous flows, the Cartesian method quickly becomes inefficient at achieving the desired anisotropic resolution. While simplex cut-cell methods alleviate this problem, current research has only been with linear background meshes, which eventually become inefficient for high Reynolds number flows around curved geometries.

\section{Implementations and Results}

\section{V.A. Finite Volume Methods}

\section{V.A.1. High-lift RANS (Venditti and Darmofal)}

Venditti and Darmofal apply output-based error estimation and mesh adaptation to a range of inviscid and viscous aerodynamic cases in two dimensions. ${ }^{29,55}$ They use a node-based, unstructured finite volume solver and solve the linear adjoint equations by time marching, similarly to the forward problem. They adapt on the remaining error in Eqn. 29 and use an average of the primal and adjoint residual localizations for the adaptive indicator. For anisotropic meshing, they use the Hessian of the Mach number to define a metric, and they remesh the domain using BAMG.

A representative example from the work of Venditti and Darmofal is that of adaptive simulation for turbulent flow over an advanced energy-efficient-transport (EET) airfoil. In this example, a sequence of lift-adapted meshes is compared to meshes adapted using only the Hessian of the Mach number with no output-error information. The resulting convergence of the lift output is shown in Figure 10a. The corrected output in both runs was calculated using the computable correction in Eqn. 29. The improved convergence of the runs adapted on the output error compared to those adapted on the Hessian is clear. The finest adapted meshes from both runs are shown in Figure 10b. Note the increased resolution of the output-adapted mesh near the main-element leading edge and over the upper surface of the main element. Also note that the Hessian-based mesh predicts the lower slat wake in a different location and does not resolve the flow in the cavity region of the main element. 


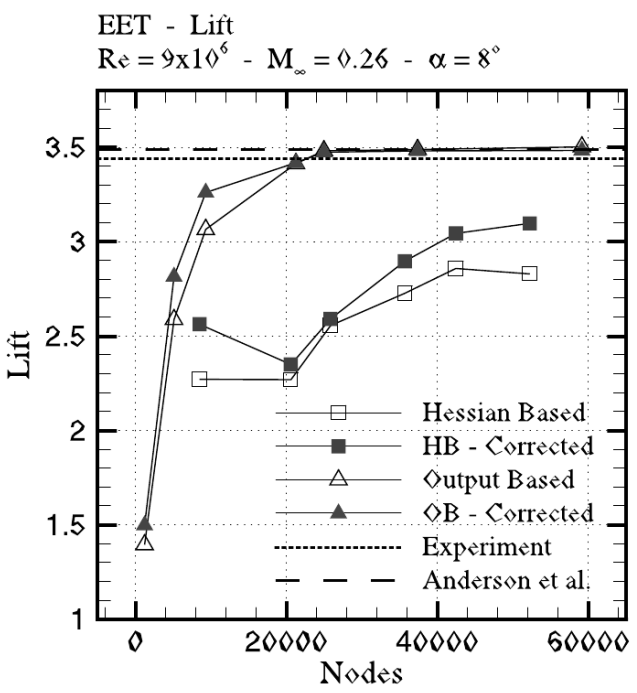

(a) Lift convergence
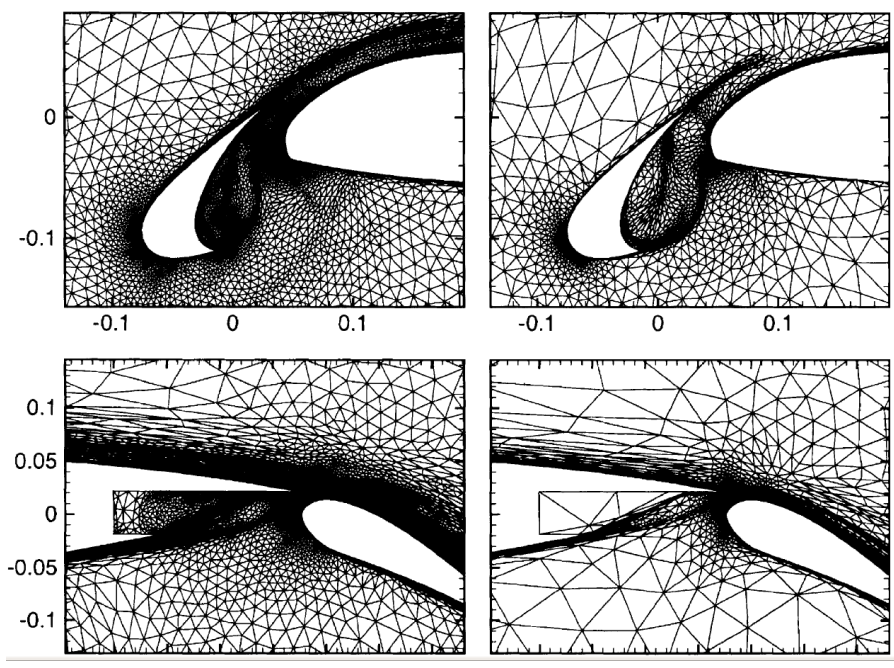

(b) Output (left) and Hessian (right) adapted meshes

Figure 10. Advanced energy-efficient-transport (EET) airfoil, $M_{\infty}=0.26, \alpha=8^{\circ}, R e=9 \times 10^{6}$. Comparison of lift convergence for output-based and Hessian-based adaptation, and near-field views of the final adapted meshes. Reproduced with permission from. ${ }^{55}$

\section{V.A.2. Launch Abort Vehicle (Nemec et al )}

Nemec et al apply an output-based adaptive framework to a Cartesian, cut-cell, finite-volume code. ${ }^{57,59}$ They solve the discrete adjoint equations by marching to steady-state with the same Runge-Kutta scheme and multigrid solver used for the flow solution. The adjoint solve requires transpose linearizations of the residual evaluation applied in reverse order, and this process is simplified by freezing the limiter used for the spatial reconstruction. Details on the adjoint implementation are given in. ${ }^{77}$

For the fine space $\mathcal{V}_{h}$ in output error estimation, Nemec et al use an embedded grid obtained by uniformly refining each hexahedral cell in the Cartesian grid. They then obtain an error indicator by weighting the residual of the coarse, linearly-reconstructed solution on the embedded grid with an adjoint error that is the difference between piecewise linear and piecewise constant reconstructions of the coarse adjoint solution. Results in ${ }^{59}$ compare the performance of this error estimate versus one that employs a more rigorous quadratic reconstruction of the adjoint and show reduced accuracy of the output error estimate but simpler implementation.

Nemec et al then define a refinement threshold error level for adaptation and at each iteration refine cells with error above this threshold, using the decreasing threshold strategy described in Section IV.C. The Cartesian hanging-node adaptation makes use of the robust cut-cell mesh generation capability in the code, ${ }^{76}$ allowing for adaptive results with complex geometries. A representative example is that of aerodynamic analysis of a Launch Abort Vehicle (LAV), illustrated in Figure 11. The output of interest for this case consists of a linear combination of the normal $(N)$ and axial $(A)$ force coefficients,

$$
J=C_{N}+0.2 C_{A},
$$

where the weight on the linear combination was determined empirically as one that yielded adequate results for both forces and moments. Note, the forces and moments are evaluated on the "metric" 
portion of the geometry, as specified in Figure 11.
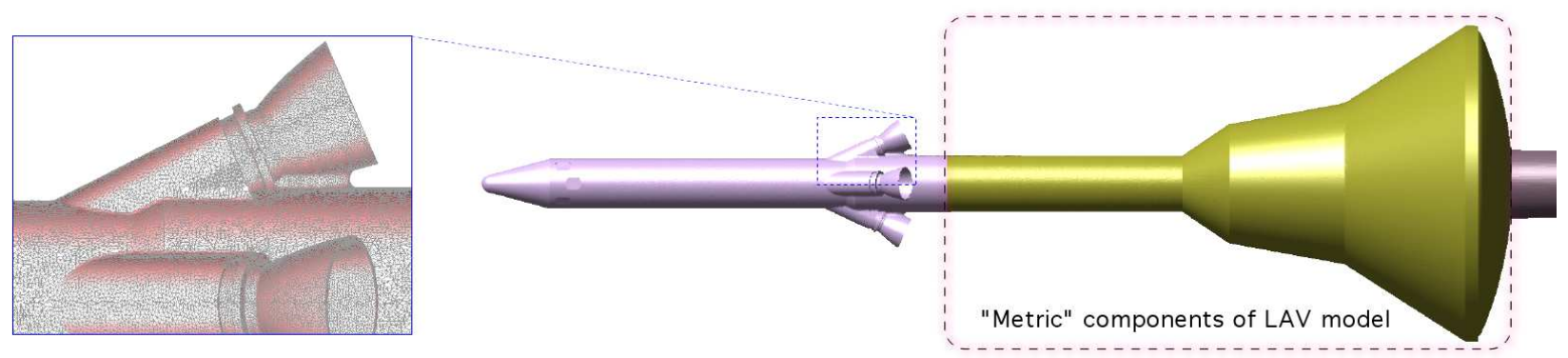

Figure 11. Definition of metric components for the LAV model. Reproduced with permission from. $^{57}$

The robustness and automation of the mesh generation process allowed Nemec et al to consider a range of Mach numbers and angles of attack. A representative case, at $M_{\infty}=1.1, \alpha=-25^{\circ}$, is shown in Figure 12. Also shown in the figure is a contour plot of the adaptive indicator, where

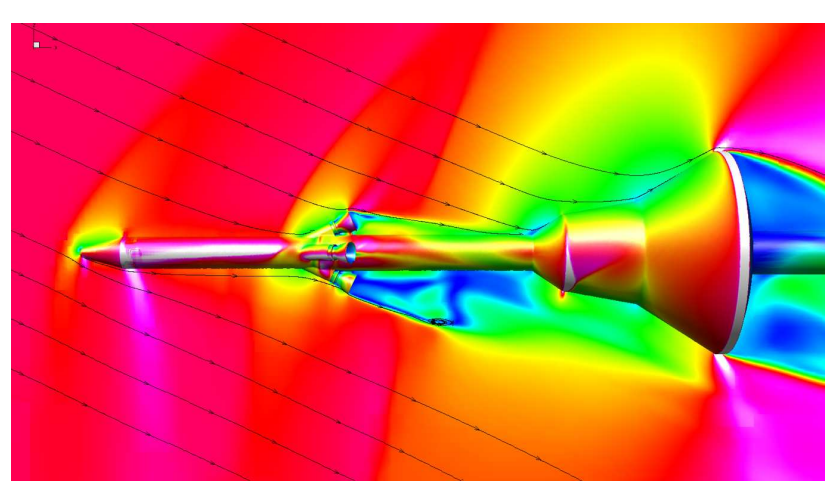

(a) Flow Solution

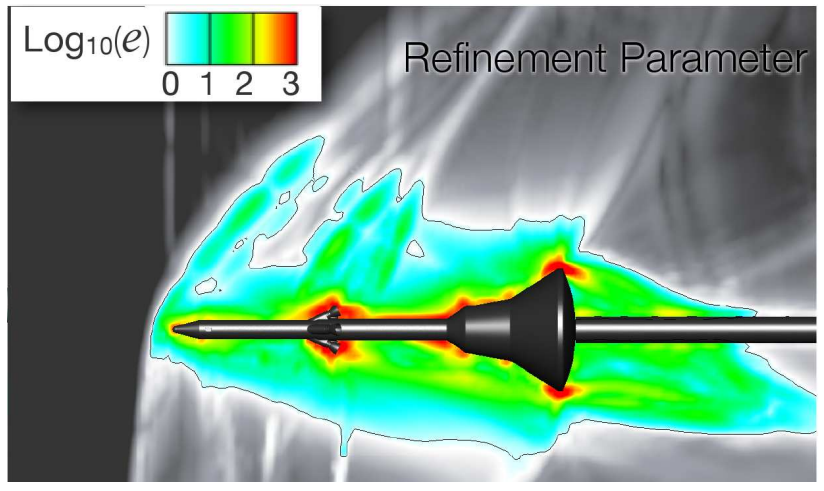

(b) Error Indicator

Figure 12. Launch Abort Vehicle (LAV) Mach number contours, $M_{\infty}=1.1, \alpha=-25^{\circ}$, and the localized error indicator. Reproduced with permission from. ${ }^{57}$

regions shown in gray-scale fall below the refinement threshold. Areas marked for refinement include the edges of the heat shield and the vicinity of the abort motors. Note that only moderate refinement is requested at the shocks, which often attract excessive refinement with heuristic feature-based indicators.

An example of a final mesh generated by the adaptive process is shown in Figure 13. As expected from the error indicator, the refinement concentrates on the edges of the heat shield and on the abort motors. The convergence of the output for this case is shown in Figure 14 on the left. Included on the same plot is the corrected output, calculated as described in Eqn. 29. The right plot in Figure 14 shows the convergence of the output error estimate. The jump in the error estimate on the final mesh is due to an incompletely-converged adjoint solution caused by the appearance of small-scale unsteadiness in the primal problem. Nevertheless, unsteady simulations on the final mesh show that the time-averaged coefficients are in good agreement with the steady results for this case. ${ }^{57}$ 


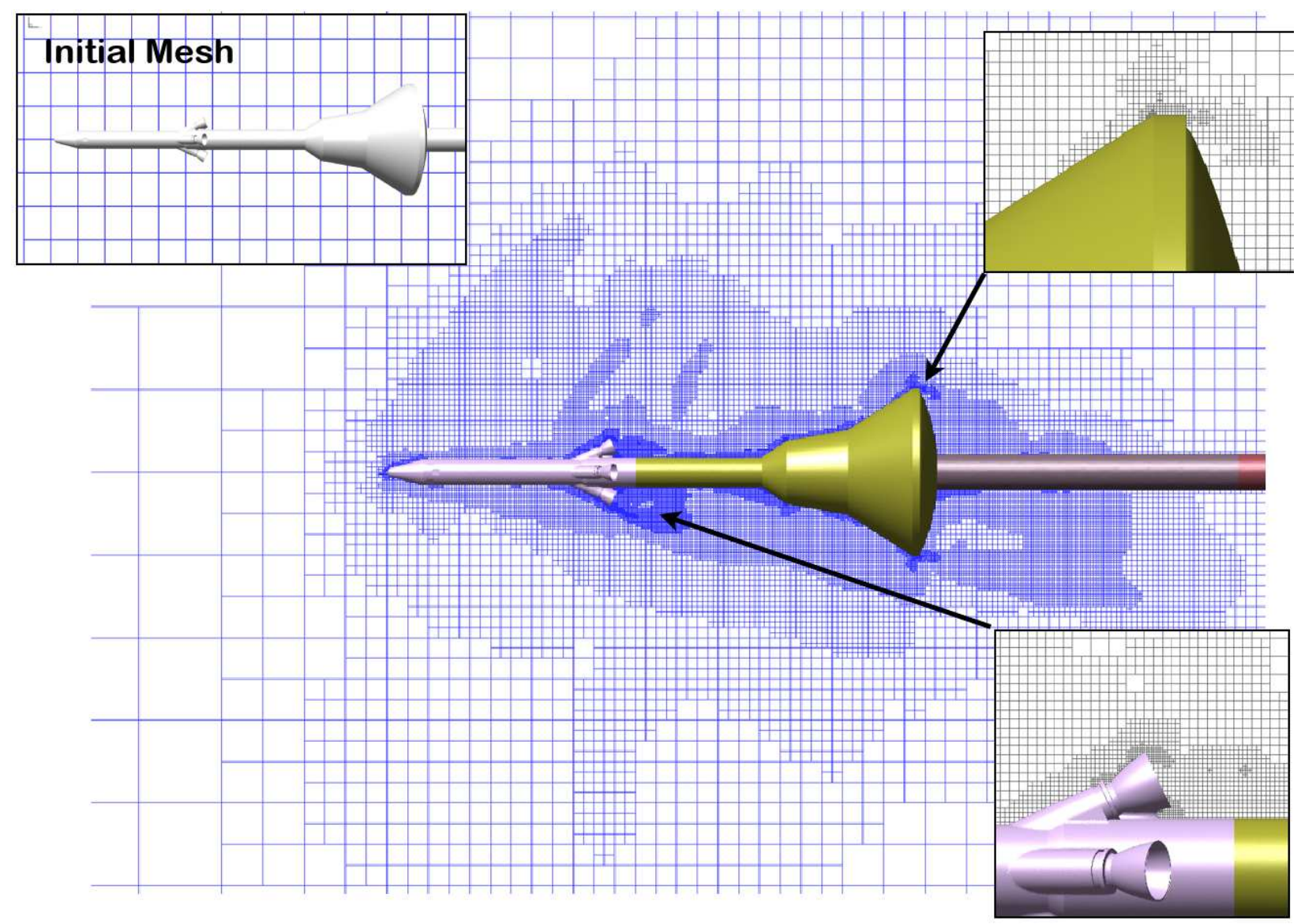

Figure 13. Initial and adapted meshes for the LAV, at $M_{\infty}=1.1, \alpha=-25^{\circ}$. The initial mesh contains 3,700 cells, while the final mesh after eight adaptation iteration contains almost two million cells. Reproduced with permission from. ${ }^{57}$
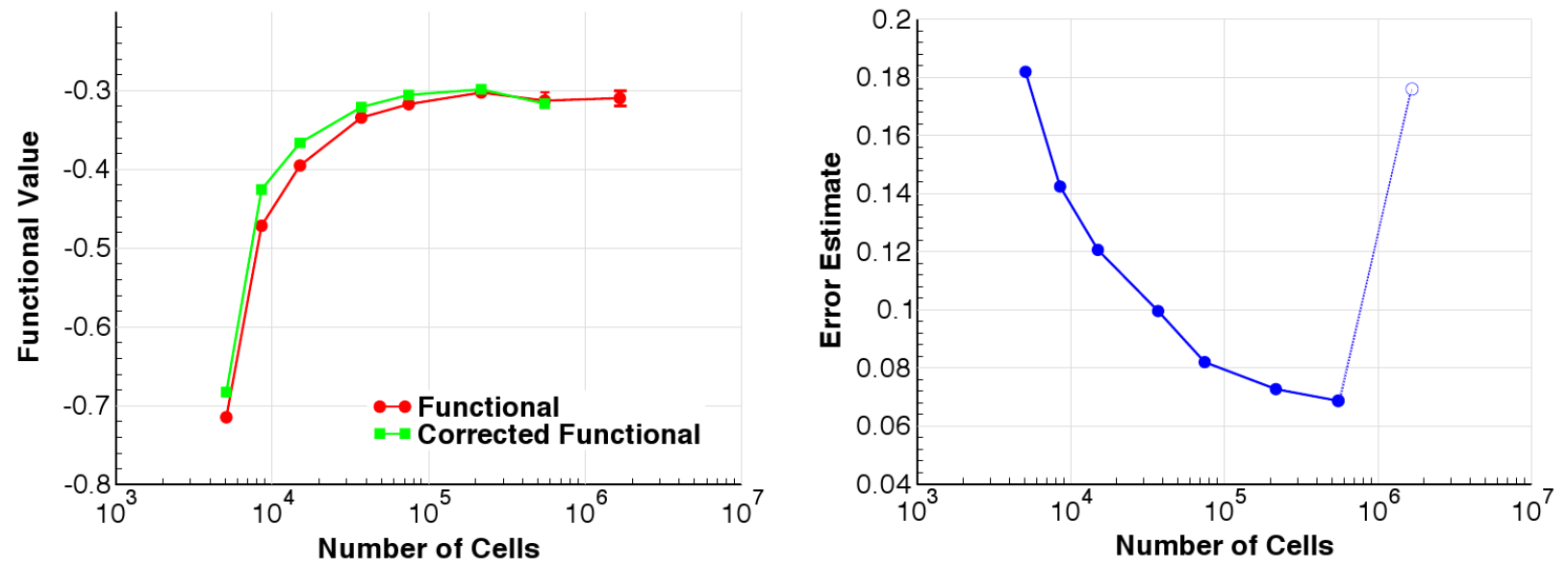

Figure 14. Output functional convergence for the LAV, at $M_{\infty}=1.1, \alpha=-25^{\circ}$. Reproduced with permission from. ${ }^{57}$ 


\section{V.A.3. Sonic Boom (Park)}

Park presents output-based, adaptive results for an unstructured, cut-cell finite volume method. ${ }^{37}$ The method is node-based, and the cut-cell approach allows for automated mesh generation. Park solves the linear adjoint equation using a dual-consistent time-marching method ${ }^{22,23}$ and adapts on the remaining error (Eqn. 29) using quadratic interpolation to obtain the fine-space solutions. He adapts on an indicator computed from the average of the localized primal and adjoint residuals. The tetrahedral grid adaptation is based on anisotropic local mesh modification operators combined with mesh movement, as described in Section IV.E.

An example case from Park's work is shown in Figure 15. The case consists of a delta wing body used in existing wind tunnel experiments, ${ }^{78}$ at $M_{\infty}=1.68, \alpha=0^{\circ}$. Of interest is the pressure signature 3.6 body lengths away from the geometry. Specifically the output consists of an integral of the square of the pressure deviation from free-stream, taken over the measurement region. The

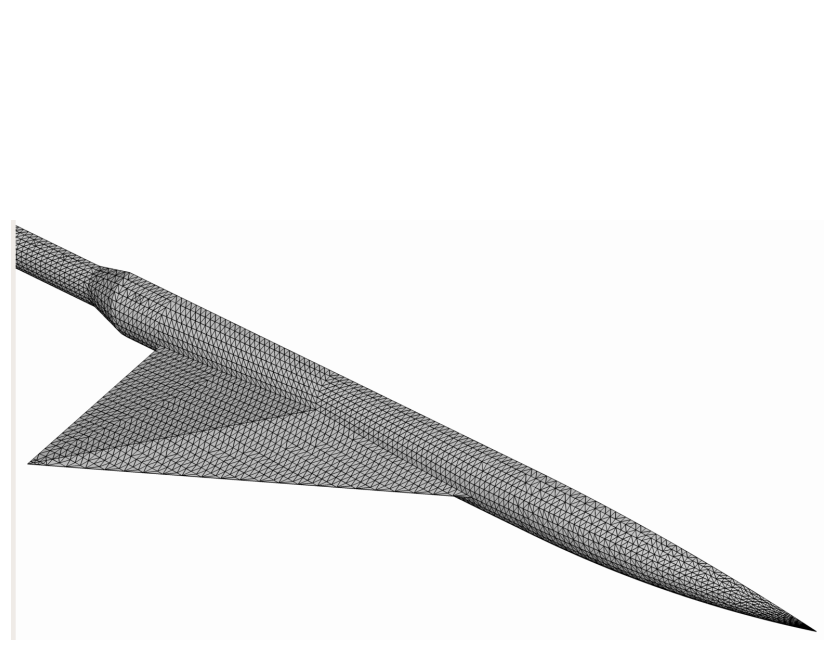

(a) Surface mesh

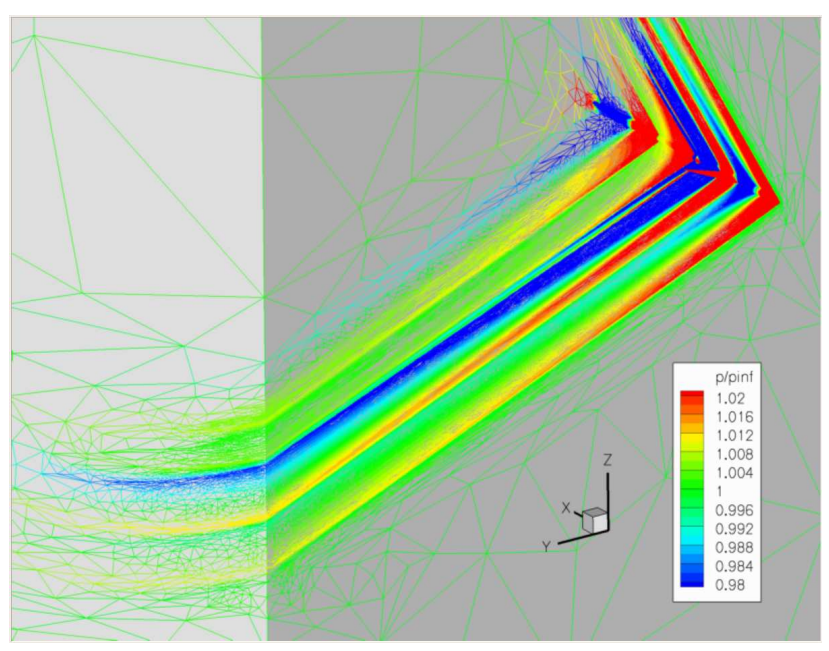

(b) Adapted background mesh

Figure 15. Delta wing-body sonic boom prediction, $M_{\infty}=1.68, \alpha=0^{\circ}$. Surface geometry mesh and an output-adapted mesh colored by pressure. Reproduced with permission from. ${ }^{37}$

triangular surface mesh in Figure 15a is the geometry representation that is used in the cut-cell method. The initial background mesh from which the geometry was cut contained 2,800 control volumes, while the final adapted background mesh in Figure 15b contained 4.9 million control volumes. Note the alignment of the cells in the final mesh with the propagated signal.

The adaptation history of the output is shown in Figure 16a, where the error bars denote the remaining error estimate. Note that the error is severely under-predicted on the very coarse initial meshes. As the shock is resolved, the error estimate becomes more accurate and begins to decrease in the latter stages of adaptation. The pressure signature 3.6 body lengths away is shown in Figure 16b. The dotted lines indicate signatures at intermediate grids during adaptation. The computed signature on the final adapted meshes agrees well with experimental data. 


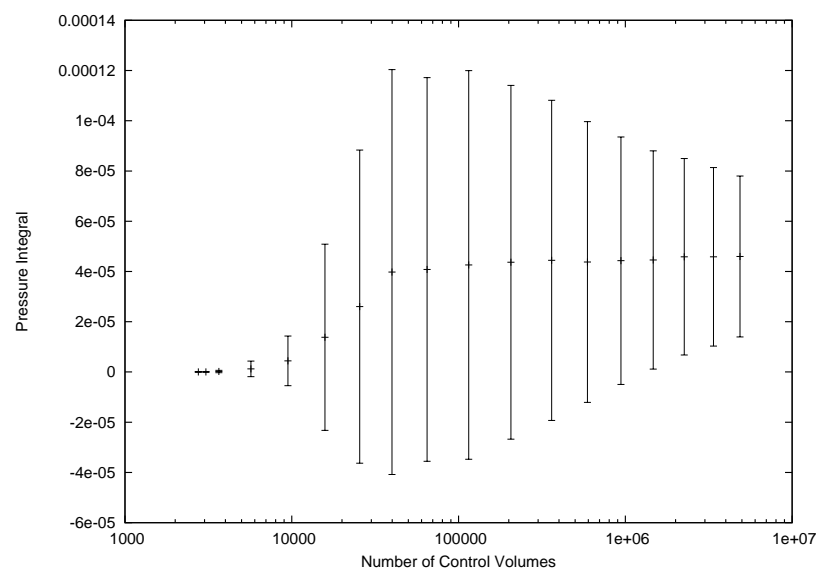

(a) Adaptation history

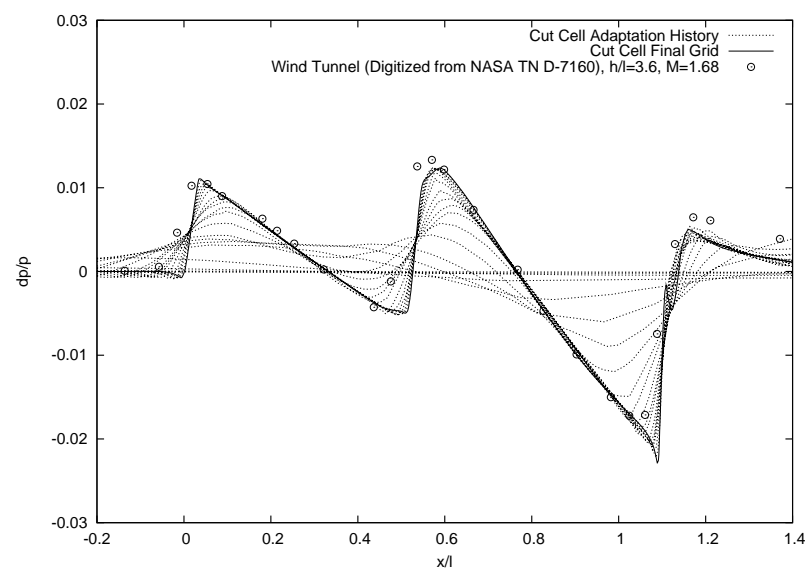

(b) Pressure signatures

Figure 16. Delta wing-body sonic boom prediction, $M_{\infty}=1.68, \alpha=0^{\circ}$. Pressure integral output history with error estimates and pressure signature convergence. Reproduced with permission from. $^{37}$

\section{V.B. Discontinuous Galerkin Methods}

\section{V.B.1. Point Error in Transonic Flow (Hartmann and Houston)}

Hartmann and Houston compare two types of error indicators for $p=1$ discontinuous Galerkin solutions of the compressible Euler equations. ${ }^{19}$ The first, type I, indicator is derived from the adjoint-weighted residual method described in this paper. The second, type II, indicator is an unweighted residual indicator, in which a priori bounds are placed on the adjoint values, eliminating the need for the adjoint solution. Adaptive results show the superior performance of using the adjoint-weighted, type I, indicator.

An example case considered by Hartmann and Houston is the converging-diverging nozzle problem shown in Figure 17. Hartmann and Houston use a damped Newton method to obtain $p=1$

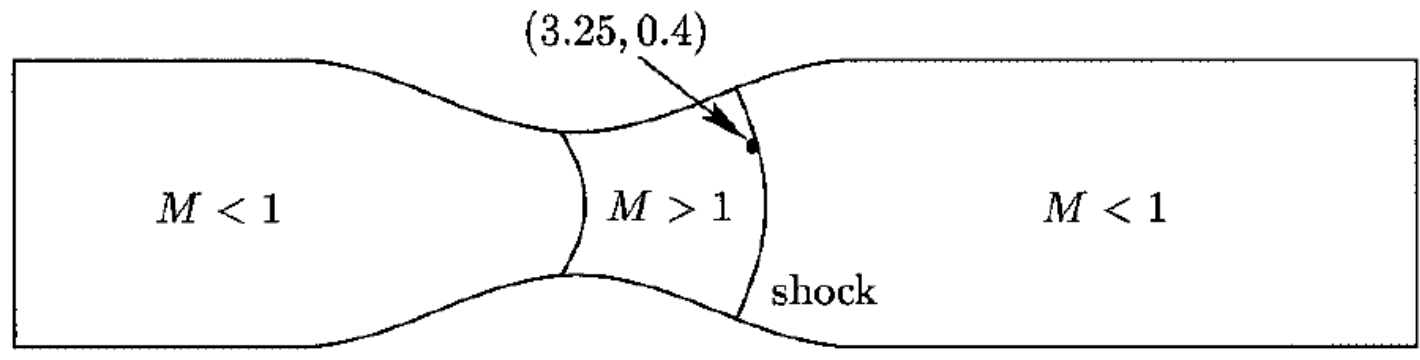

Figure 17. Converging-diverging nozzle geometry. The output of interest is the density immediately before the shock. Reproduced with permission from. ${ }^{19}$

primal and adjoint solutions on a geometry represented by quadratic $(q=2)$ elements. As the fine space for error estimation, they use order $p=2$ interpolation on the same mesh. They adapt the quadrilateral meshes based on the indicators with fixed-fraction, hanging-node refinement and coarsening. Figure 18 shows adapted meshes for the two types of error indicators. The type II error indicator, which does not use the adjoint solution, refines mainly the region near the shock. On 
the other hand, the type I error indicator leads to refinement along the characteristics upstream of the point of interest. This targeted refinement yields a lower output error with fewer degrees of freedom compared to the type II refinement.

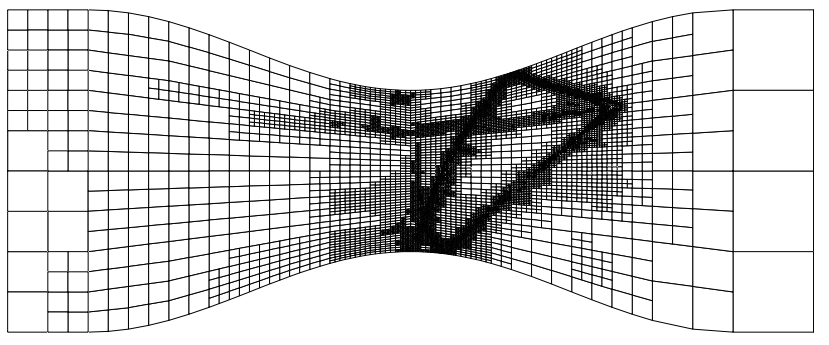

(a)

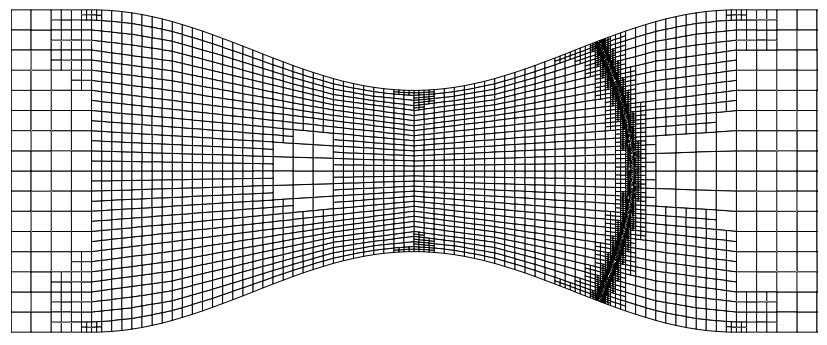

(b)

Figure 18. Converging-diverging nozzle: (a) mesh adapted using the type I error indicator, based on the adjoint-weighted residual, with 172,880 degrees of freedom and an output error of $6.947 \times 10^{-6}$. (b) mesh adapted using the type II error indicator, with 341,648 degrees of freedom and an output error of $2.842 \times 10^{-5}$. Reproduced with permission from. ${ }^{19}$

\section{V.B.2. Hypersonic Heat Transfer (Barter and Darmofal)}

Barter and Darmofal apply output-based error estimation and mesh adaptation to discontinuous Galerkin solutions containing shocks, using a discretization stabilized with smooth artificialviscosity. ${ }^{28,79}$ In this discretization, the artificial viscosity on each element is interpolated with the same polynomial basis as the state, and the coefficients for these polynomials are agglomerated into the unknown state vector. The necessary additional equations are obtained by discretizing a diffusion partial differential equation for the artificial viscosity.

Barter and Darmofal use several sweeps of a block-Jacobi smoother in order to approximate $\boldsymbol{\psi}_{h}^{\mathrm{mv}}$ on a space enriched to order $p+1$. As in related work, the adaptive indicator is formed by averaging localized primal and adjoint output error estimates.

The artificial viscosity stabilization enables Barter and Darmofal to adaptively solve transonic, supersonic, and hypersonic flow problems. A representative example is that of hypersonic flow at $M_{\infty}=17.605, R e=376,930$ over a cylinder geometry, also studied in previous work. ${ }^{80}$ The problem setup and the initial mesh for adaptation are shown in Figure 19. The output used for adaptation is the integrated heat flux to the cylinder, non-dimensionalized to form the average Stanton number on the surface,

$$
Q_{\text {wall }}=\int q_{\text {wall }} d s ; \quad \overline{C_{h}}=\frac{\frac{1}{\pi R} Q_{\text {wall }}}{c_{p} \rho_{\infty} V_{\infty}\left(T_{t, \infty}-T_{\text {wall }}\right)} .
$$

BAMG is used as the mesh generator for the metric-driven adaptation, where an anisotropy metric is defined using the Mach number interpolation error. To create meshes with curved $(q=3)$ anisotropic elements for the thin boundary layer around the cylinder, Barter and Darmofal perform adaptation in a mapped, rectangular space, transforming the requested metric appropriately as described in. ${ }^{16}$ Figure 20 shows the final adapted mesh for $p=2$ and the output convergence history compared to uniform refinement. The final adapted mesh exhibits refinement in the bow shock, but only to the extent that it impacts the heat flux on the cylinder. Refinement is also seen in the stagnation streamline, an area to which the heat flux output is highly sensitive. The 


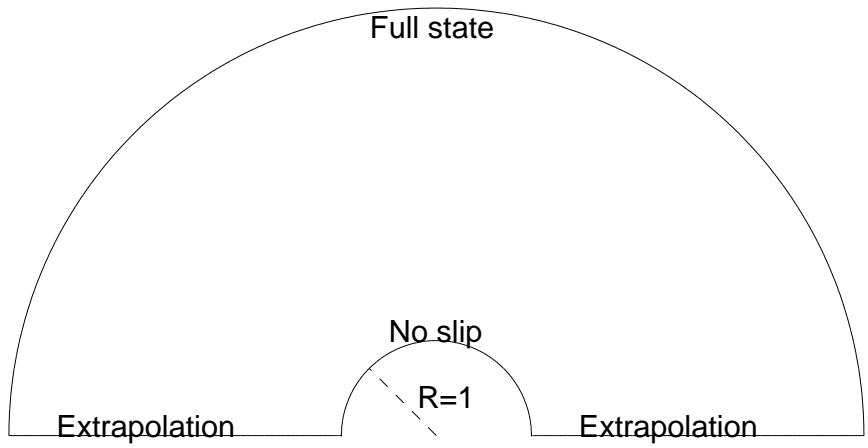

(a) Domain and BCs

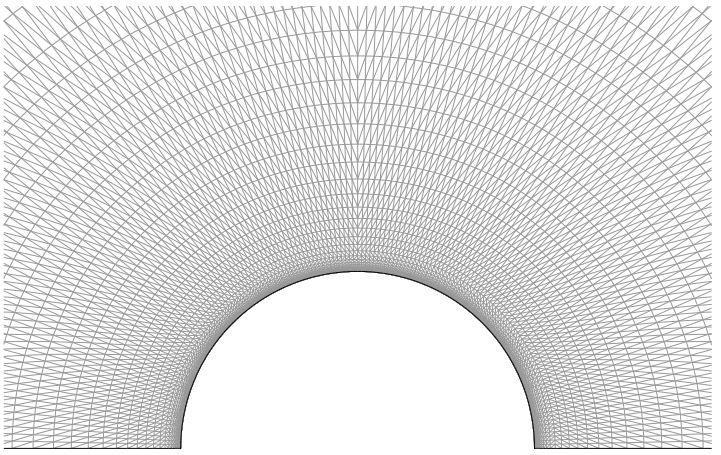

(b) Initial mesh

Figure 19. Hypersonic flow over a 2D half-cylinder at $M_{\infty}=17.605, R e=376,930$ : domain with boundary conditions and initial mesh for adaptation. Note, flow is from top to bottom. Reproduced with permission from. ${ }^{28}$

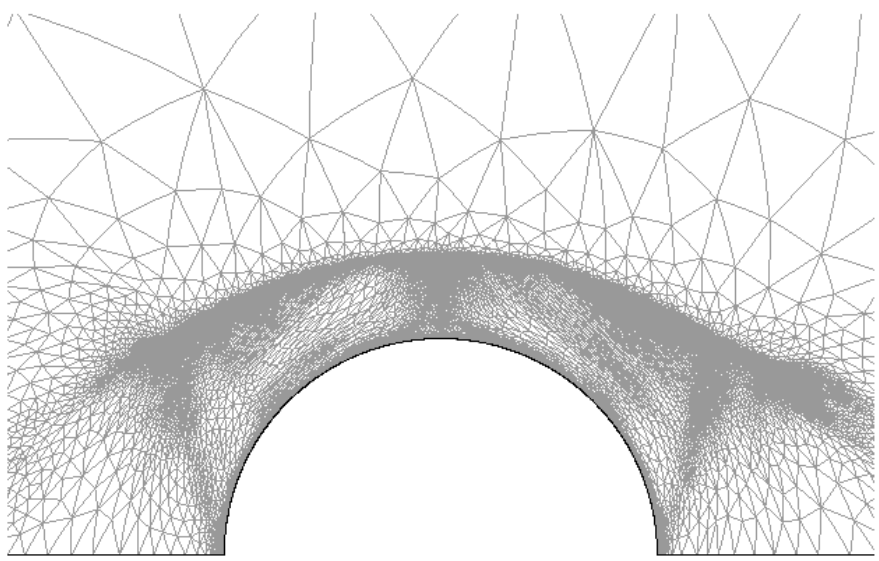

(a) Adapted mesh, $p=2$

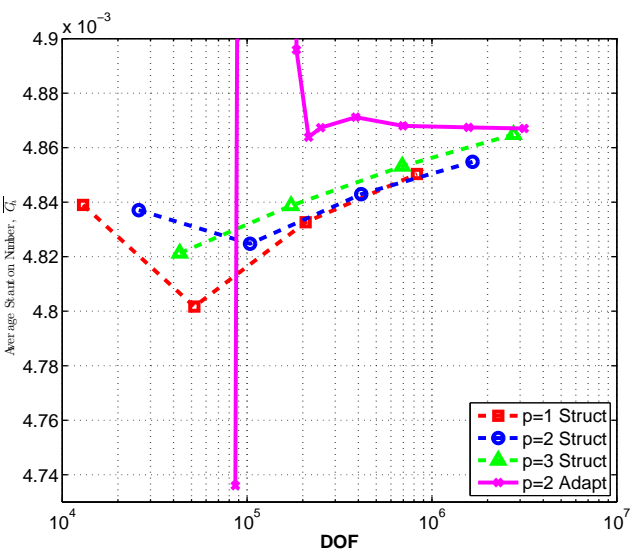

(b) Output convergence

Figure 20. Hypersonic flow over a 2D half-cylinder at $M_{\infty}=17.605, R e=376,930$ : adaptation on average surface Stanton number. Final adapted mesh for $p=2$ and output convergence for $p=2$ adaptation ("Adapt") compared to uniform refinement at orders $p=1,2,3$ ("Struct"). Reproduced with permission from. ${ }^{28}$ 
convergence history shows that the adapted run converges to an output within 0.02 percent of the final value with approximately three million degrees of freedom.

\section{V.B.3. Laminar Flows using Simplex Cut Cells (Fidkowski and Darmofal)}

Fidkowski and Darmofal use an output-based adaptive indicator to drive cut-cell $h$-adaptation for a high-order discontinuous Galerkin discretization of the compressible Navier-Stokes equations. ${ }^{36,63}$ They use an implicit solution procedure for the primal problem that stores the full, compact-stencil, residual linearization, so that the discrete adjoint solution requires only one transpose application of the same linear solver. The fine-space adjoint solution $\boldsymbol{\psi}_{h}^{\mathrm{mv}}$ is approximated by reconstructing the coarse adjoint, $\boldsymbol{\psi}_{H}$, on the same mesh with order enriched to $p+1$. The adaptive indicator is formed by averaging localized primal and adjoint output error estimates.

The cut-cell method, illustrated in Figure 21, employs simplex elements and metric-driven global re-meshing of the background domain to enable automated and anisotropic mesh adaptation. Meshing is performed using BAMG with anisotropy based on interpolation error in the Mach number. Fidkowski and Darmofal apply the cut-cell adaptive method to several inviscid and viscous

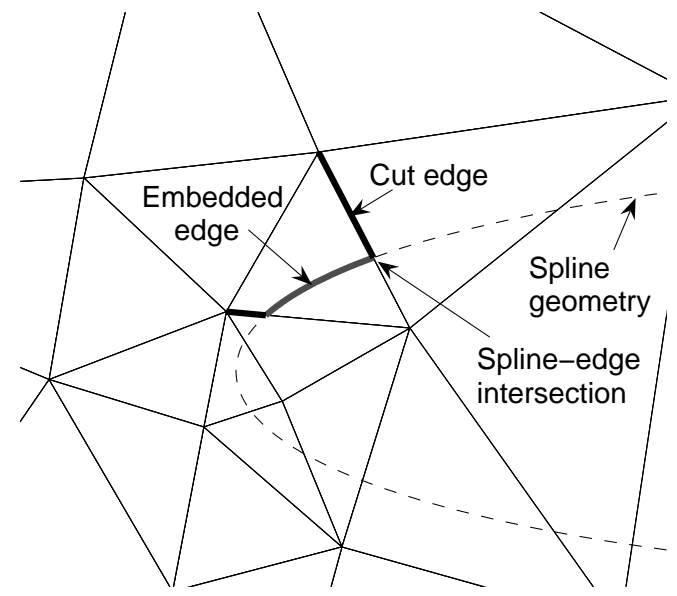

Figure 21. Illustration of the simplex cut-cell method used in ${ }^{63}$ for $h$-adaptation on high-order discontinuous Galerkin solutions. Curved cut cells are obtained by intersecting a cubic spline geometry representation with a triangular background mesh generated using BAMG. High order integration rules are derived on the resulting irregularly-shaped cut cells.

flows. A representative example is that of viscous flow around a NACA 0012 airfoil at $M_{\infty}=0.5$, $\alpha=2^{\circ}, R e=5,000$. Drag-adapted meshes for cubic, $p=3$, solution interpolation are shown in Figure 22 for both the cut-cell method and a boundary-conforming method that requires curving of boundary elements and is consequently not as robust. In these meshes, areas of refinement include the boundary layer, a large extent of the wake, and, to a lesser extent, the flow in front of the airfoil.

Figure 23 compares adaptive convergence histories of the drag error for interpolation orders $p=1,2,3$. The boundary-conforming and cut-cell runs converge to the same drag value, and the histories are similar. In both sets of runs, $p=3$ requires only slightly fewer degrees of freedom than $p=2$, while $p=1$ remains the most expensive. 

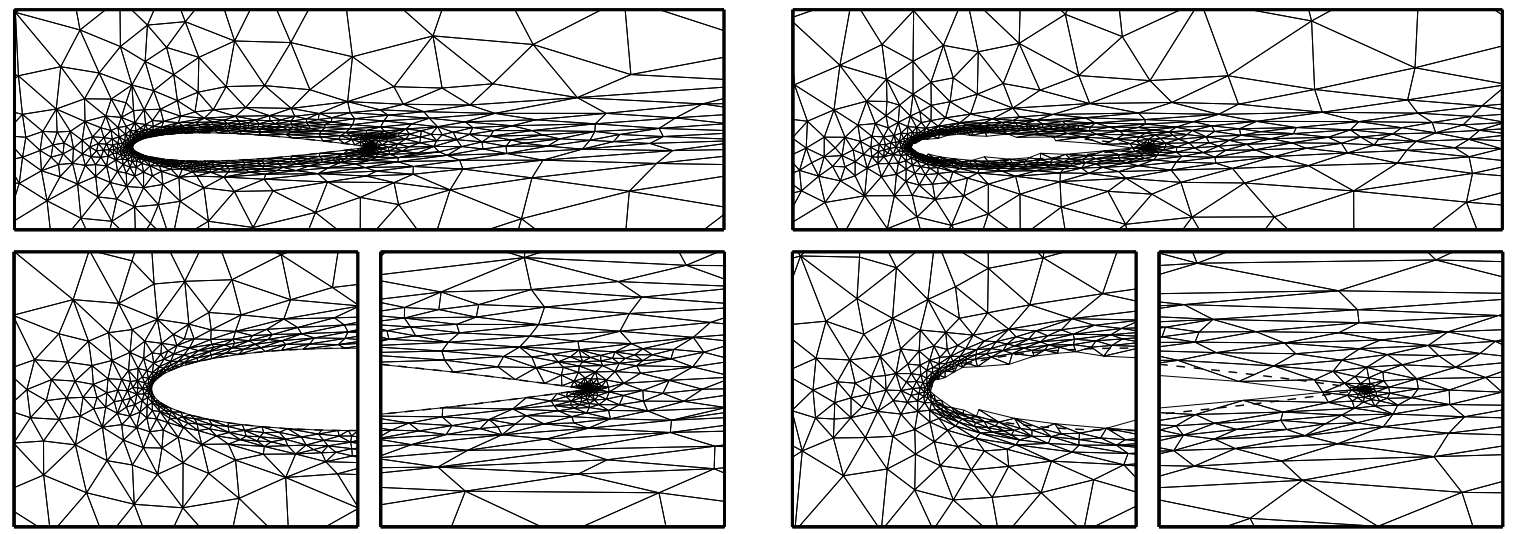

(a) Boundary-conforming: 1929 elements

(b) Cut-cell: 1840 elements

Figure 22. NACA $0012 M_{\infty}=0.5, \alpha=2^{\circ}, R e=5,000, p=3$ interpolation. Final boundaryconforming and cut-cell meshes adapted on drag. ${ }^{63}$

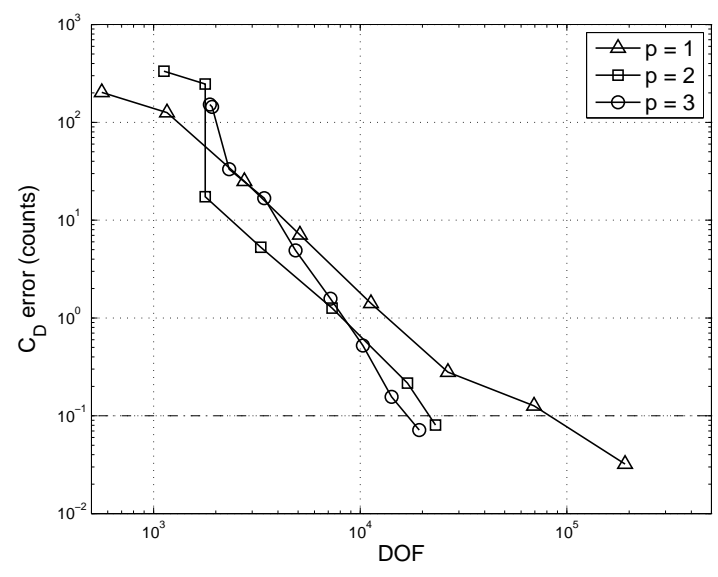

(a) Boundary-conforming

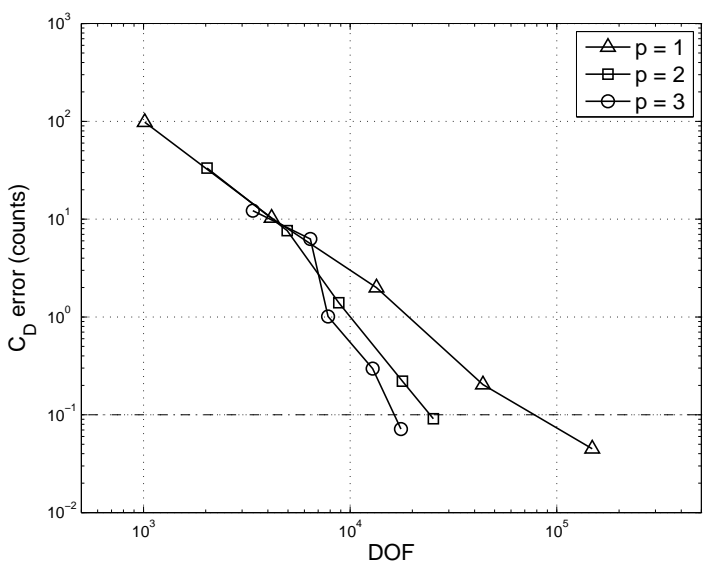

(b) Cut-cell

Figure 23. NACA $0012 M_{\infty}=0.5, \alpha=2^{\circ}, R e=5,000$, Drag error versus degrees of freedom for interpolation orders $p=1,2,3$. Dashed line indicates prescribed tolerance of 0.1 drag counts. ${ }^{63}$ 


\section{Challenges and Ongoing Research}

\section{VI.A. Robust Mesh Adaptation}

Performing mesh adaptation robustly and efficiently for complex three-dimensional configurations is still a challenge and an area of ongoing research. The lack of robust and efficient mesh adaptation is probably the largest barrier limiting the application of output-based adaptation to simple geometries and/or simplified physics (i.e. inviscid flows as opposed to viscous flows).

The main robustness issue occurs during boundary point insertion on curved geometries: maintaining geometry fidelity can lead to invalid, negative-volume, elements on the interior, especially for highly-anisotropic meshes. ${ }^{48}$ Currently, resolution of such situations is often attempted with iterative application of local operators and local re-meshing. ${ }^{81}$ However, guaranteed geometric fidelity and element validity in highly anisotropic meshes around curved geometries has yet to be demonstrated.

Efficient adaptation refers to using available degrees of freedom as effectively as possible. A notable example of a situation in which efficient adaptation is important is in the resolution of curved, anisotropic, solution features, as illustrated in Figure 24. Simple isotropic mesh adaptation in these

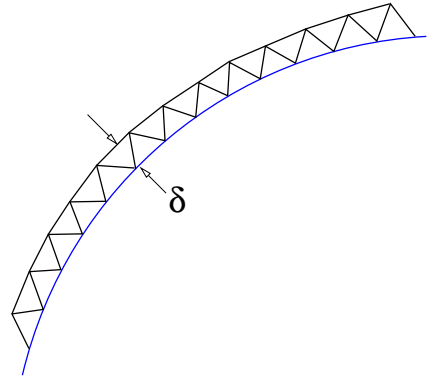

(a) Isotropic

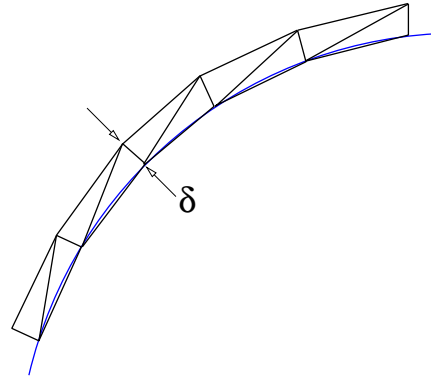

(b) Linear anisotropic

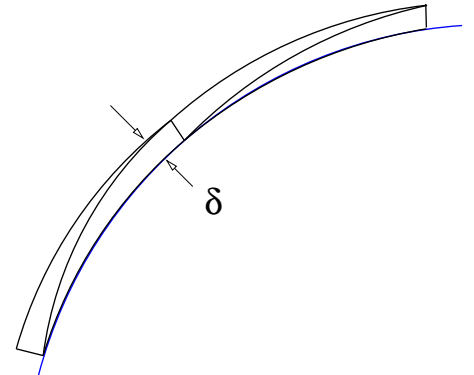

(c) Curved anisotropic

Figure 24. Resolution of a thin, curved, anisotropic, two-dimensional layer using various mesh adaptation strategies. A resolution length of $\delta$ is required normal to the feature, whereas the solution does not change much tangentially to the feature. Stretching elements to increase their aspect ratio, defined as the ratio of the largest to smallest element diameters, improves efficiency of the mesh, measured in terms of degrees of freedom required to resolve the feature. Additionally curving the elements further improves efficiency by alleviating the curvatureimposed geometry resolution constraint.

areas, Figure 24a, wastes resolution in directions where the solution is not changing significantly. In three dimensions, the additional degrees of freedom in the two directions where the solution is not changing quickly make resolution of thin layers impractical. Stretching elements along these directions helps significantly, especially when the anisotropic layer is approximately straight. When the layer is curved, however, the maximum feasible aspect ratio of linear stretched elements is bounded by the curvature of the layer since the geometry must be resolved, as illustrated in Figure 24b. Efficient resolution of very thin and curved features, such as those encountered in Reynolds-Averaged Navier-Stokes boundary layers, must therefore employ curved elements, as shown in Figure 24c.

Robust boundary-conforming mesh generation and adaptation techniques currently exist for many applications requiring only isotropic meshes, e.g. ${ }^{82}$ An exception here is mesh generation for high-order methods that require curved elements on geometry boundaries, although in the 
isotropic case, node movement and certain heuristics are generally sufficient. However, robust boundary-conforming meshing and adaptation for three-dimensional anisotropic solution features are still areas of ongoing research. Curving elements for improved efficiency adds another layer of complexity, especially when curved anisotropic features occur away from the geometry.

Cut-cell techniques eliminate the requirement that the mesh needs to respect the geometry boundary, and can be robust for complex geometries. However, existing Cartesian methods only allow for isotropic refinement, except in special cases when the features are aligned with the mesh. ${ }^{57,76}$ Simplex cut-cell methods allow for element stretching, and hence resolution with improved efficiency. ${ }^{63}$ However, additional research is required to extend these methods to stretch and curve elements so as to handle curved anisotropic features in three dimensions.

\section{VI.B. Computable Error Bounds}

The output error estimate in Eqns. 21 and 25 is not a bound for the true output error in the discrete solution because of the approximations made in the derivation and because of the use of a finite-dimensional fine space, $\mathcal{V}_{h}$. If the computational mesh is very coarse, the fine space obtained by uniform mesh refinement or interpolation order increase may still be too coarse to faithfully resolve the output of interest. In such a case, the output error estimate may be severely unreliable.

An example of this effect is the pressure signature adaptation on the delta wing-body sonic boom case considered by Park, as described in Section V.A.3. The vertical bars in Figure 16a show the pressure integral error estimate at each adaptation iteration. The relatively small size of the error bars in the first few iterations indicates that the output error is severely under-predicted on the first meshes. On these meshes and the fine spaces derived from them, the sonic boom signature is not at all resolved, and the output error estimate is meaningless. The estimate only becomes accurate after six or seven adaptation iterations, which corresponds to a substantial increase in the number of degrees of freedom. It does not start dropping until about ten adaptation iterations. The risk of an inaccurate error estimate on coarse meshes is that an automated adaptive process may terminate early, without sufficiently resolving the output of interest.

There exists a body of research that addresses this risk through the computation of error bounds on the outputs of interest. ${ }^{83-88}$ The goal of this research has been to derive strict, constantfree, lower and upper bounds for outputs of interest. The bounds calculations are based on a reformulation of the output calculation into a constrained minimization problem with a convex objective function, with the model equations entering the problem as equality constraints. Initially,

the bounds calculations were strict with respect to a conservatively refined computational mesh; ${ }^{83-85}$ more recently the calculations have been extended so that the bounds are strict with respect to the exact weak solution of the partial differential equation. ${ }^{86-88}$ These calculations rely on the solution of a local dual problem that transforms an infinite-dimensional minimization problem into a finite-dimensional feasibility problem. ${ }^{86}$

This strategy has been applied to symmetric and non-symmetric coercive problems, ${ }^{83,86}$ certain constrained and non-coercive problems, ${ }^{84}$ and also to problems with nonlinear outputs and equations. ${ }^{85,89,90}$ For nonlinear problems, the present procedure yields bounds only for sufficiently resolved meshes, where the required mesh resolution is not known a priori ${ }^{85}$

The bound gap, which is the difference between the upper and lower bounds on the output, can be separated into positive contributions from each element, yielding an indicator for mesh adaptation. A strategy that refines elements with a large contribution to the bound gap will efficiently yield a tighter estimate of the output. The result of applying such a strategy is a 
solution with fully certifiable precision of integrated outputs. Additional research in this area is necessary to extend the bounds computations to additional equation sets, especially for aerospace Computational Fluid Dynamics applications.

\section{VI.C. Unsteady Applications}

As output-based adaptation is applied to increasingly complex flows, problems that include unsteadiness will naturally arise. Even for applications targeting nominally steady solutions, unsteadiness is likely to occur as wakes are resolved with adaptation. The work of Nemec et al described in Section V.A.2 is an example of this occurring.

Existing work in the application of adjoint sensitivity analysis to unsteady problems comes largely from shape optimization research, and ranges from frequency domain methods for periodic unsteady flows ${ }^{91,92}$ to time-accurate continuous and discrete adjoint methods. ${ }^{93-97}$ Incorporation of time dependence in adjoint analysis is theoretically a well understood problem. However, the algorithmic issues involved in solving an unsteady adjoint are substantial as the unsteady adjoint must be marched backward in time from the final to the initial state. For nonlinear problems, the solution time history must be stored or reconstructed to build the required Jacobians and output linearizations at each time level.

Mani and Mavriplis apply the adjoint-weighted residual technique to drive time step adaptation in unsteady flow simulations with deforming meshes. ${ }^{96}$ For time adaptation, the time step is allowed to vary, but the spatial mesh distribution is not changed (except for prescribed deformation). The output error is thus calculated by evaluating the residual of the unsteady primal solution on a finer temporal discretization and weighting it with the adjoint solution on that discretization. The results in Mani and Mavriplis's work indicate a computational savings over uniform temporal refinement for smooth unsteady problems. An area of future research is combined spatial and temporal adaptation for problems exhibiting non-smooth spatial and temporal features, where the computational savings of an output-based adaptive method could be very significant.

\section{Acknowledgments}

The authors would like to acknowledge M. Aftosmis, G. Barter, R. Hartmann, P. Houston, D. Mavriplis, M. Nemec, T. Oliver, and M. Park, for permission to present their results and to use their figures. In addition the authors gratefully acknowledge the support of the Massachusetts Institute of Technology and the University of Michigan.

\section{References}

\footnotetext{
${ }^{1}$ Levy, D. W., Zickuhr, T., Vassberg, J., Agrawal, S., Wahls, R. A., Pirzadeh, S., and Hemsch, M. J., "Data summary from the First AIAA Computational Fluid Dynamics Drag Prediction Workshop," Journal of Aircraft, Vol. 40, No. 5, 2003, pp. 875-882.

${ }^{2}$ Laflin, K. R., Vassberg, J. C., Wahls, R. A., Morrison, J. H., Brodersen, O., Rakowitz, M., Tinoco, E. N., and Godard, J.-L., "Summary of data from the second AIAA CFD drag prediction workshop," AIAA Paper 2004-0555, 2004.

${ }^{3}$ Frink, N. T., "Test case results from the 3rd AIAA drag prediction workshop," NASA Langley, 2007, http://aaac.larc.nasa.gov/tsab/cfdlarc/aiaa-dpw/ Workshop3/final_results_jm.tar.gz.

${ }^{4}$ Vassberg, J. C., DeHaan, M. A., and Sclafani, T. J., "Grid generation requirements for accurate drag predictions based on OVERFLOW calculations," AIAA Paper 2003-4124, 2003.

${ }^{5}$ Fidkowski, K. J., A Simplex Cut-Cell Adaptive Method for High-Order Discretizations of the Compressible Navier-Stokes Equations, Ph.D. thesis, Massachusetts Institute of Technology, Cambridge, Massachusetts, 2007.
} 
${ }^{6}$ Morrison, J. H. and Hemsch, M. J., "Statistical analysis of CFD solutions from the third AIAA drag prediction workshop," AIAA Paper 2007-254, 2007.

${ }^{7}$ Mavriplis, D. J., "Results from the 3rd drag prediction workshop using the NSU3D unstructured mesh solver," AIAA Paper 2007-256, 2007.

${ }^{8}$ Verfürth, R., "A posteriori error estimation and adaptive mesh-refinement techniques," Journal of Computational and Applied Mathematics, Vol. 50, 1994, pp. 67-83.

${ }^{9}$ Ainsworth, M. and Oden, J. T., "A posteriori error estimation in finite element analysis," Computer Methods in Applied Mechanics and Engineering, Vol. 142, 1997, pp. 1-88.

${ }^{10}$ Becker, R. and Rannacher, R., "An optimal control approach to a posteriori error estimation in finite element methods," Acta Numerica, edited by A. Iserles, Cambridge University Press, 2001, pp. 1-102.

${ }^{11}$ Giles, M. and Pierce, N., "Adjoint error correction for integral outputs," Lecture Notes in Computational Science and Engineering: Error Estimation and Adaptive Discretization Methods in Computational Fluid Dynamics, Vol. 25, Springer, Berlin, 2002.

${ }^{12}$ Giles, M. B. and Süli, E., "Adjoint methods for PDEs: a posteriori error analysis and postprocessing by duality," Acta Numerica, Vol. 11, 2002, pp. 145-236.

${ }^{13} \mathrm{Lu}$, J., An a Posteriori Error Control Framework for Adaptive Precision Optimization Using Discontinuous Galerkin Finite Element Method, Ph.D. thesis, Massachusetts Institute of Technology, Cambridge, Massachusetts, 2005.

${ }^{14}$ Giles, M. B. and Pierce, N. A., "Adjoint equations in CFD: duality, boundary conditions and solution behavior," AIAA Paper 97-1850, 1997.

${ }^{15}$ Hartmann, R., "Adjoint consistency analysis of discontinuous Galerkin discretizations," SIAM Journal on Numerical Analysis, Vol. 45, No. 6, 2007, pp. 2671-2696.

${ }^{16}$ Oliver, T. A., A High-Order, Adaptive, Discontinuous Galerkin Finite Elemenet Method for the ReynoldsAveraged Navier-Stokes Equations, Ph.D. thesis, Massachusetts Institute of Technology, Cambridge, Massachusetts, 2008.

${ }^{17}$ Arnold, D. N., Brezzi, F., Cockburn, B., and Marini, L. D., "Unified analysis of discontinuous galerkin methods for elliptic problems," SIAM Journal on Numerical Analysis, Vol. 39, No. 5, 2002, pp. 1749-1779.

${ }^{18}$ Pierce, N. A. and Giles, M. B., "Adjoint recovery of superconvergent functionals from PDE approximations," SIAM Review, Vol. 42, No. 2, 2000, pp. 247-264.

${ }^{19}$ Hartmann, R. and Houston, P., "Adaptive discontinuous Galerkin finite element methods for the compressible Euler equations," Journal of Computational Physics, Vol. 183, No. 2, 2002, pp. 508-532.

${ }^{20}$ Barth, T. and Larson, M., "A posteriori error estimates for higher order Godunov finite volume methods on unstructured meshes," Finite Volumes for Complex Applications III, edited by R. Herban and D. Kröner, Hermes Penton, London, 2002, pp. 41-63.

${ }^{21} \mathrm{Lu}$, J. and Darmofal, D. L., "A quasi-minimal residual method for simultaneous primal-dual solutions and superconvergent functional estimates," SIAM Journal on Scientific Computing, Vol. 24, No. 5, 2003, pp. 1693-1709.

${ }^{22}$ Giles, M. B., Duta, M. C., Müller, J.-D., and Pierce, N. A., "Algorithm developments for discrete adjoint methods," AIAA Journal, Vol. 41, No. 2, 2003, pp. 198-205.

${ }^{23}$ Nielsen, E. J., Lu, J., Park, M. A., and Darmofal, D. L., "An implicit, exact dual adjoint solution method for turbulent flows on unstructured grids," Computers and Fluids, Vol. 33, 2004, pp. 1131-1155.

${ }^{24}$ Zhang, X. D., Vallet, M.-G., Dompierre, J., Labbe, P., Pelletier, D., Trepanier, J.-Y., Camarero, R., Lassaline, J. V., Manzano, L. M., and Zingg, D. W., "Mesh adaptation using different error indicators for the Euler equations," AIAA Paper 2001-2549, 2001.

${ }^{25}$ Houston, P. and Süli, E., "Error estimation and adaptive discretization methods in computational fluid dynamics," Error Estimation and Adaptive Discretization Methods in Computational Fluid Dynamics, edited by T. Barth and H. Deconinck, Springer-Verlag, Heidelberg, Lecture Notes in Computational Science and Engineering Vol 25, 2002, pp. 269-344.

${ }^{26}$ Solín, P. and Demkowicz, L., "Goal-oriented hp-adaptivity for elliptic problems," Computer Methods in Applied Mechanics and Engineering, Vol. 193, 2004, pp. 449-468.

${ }^{27}$ Rannacher, R., "Adaptive Galerkin finite element methods for partial differential equations," Journal of Computational and Applied Mathematics, Vol. 128, 2001, pp. 205-233.

${ }^{28}$ Barter, G. E., Shock Capturing with PDE-Based Artificial Viscosity for an Adaptive Higher-Order Discontinuous Galerkin Finite Element Method, Ph.D. thesis, Massachusetts Institute of Technology, Cambridge, Massachusetts, 2008.

${ }^{29}$ Venditti, D. A. and Darmofal, D. L., "Grid adaptation for functional outputs: application to two-dimensional inviscid flows," Journal of Computational Physics, Vol. 176, No. 1, 2002, pp. 40-39.

${ }^{30}$ Szabo, B. A., "Estimation and control of error based on $p$ convergence," Accuracy Estimates and Adaptive Refinements in Finite Element Computations, edited by I. Babuska, O. C. Zienkiewicz, J. Gago, and E. R. de Oliveira, John wiley \& Sons Ltd., 1986, pp. 61-78. 
${ }^{31}$ Rachowicz, W., Demkowicz, L., and Oden, J., "Toward a universal $h-p$ adaptive finite element strategy, part 3. design of $h-p$ meshes." Computer Methods in Applied Mechanics and Engineering, Vol. 77, 1989, pp. 181-212.

${ }^{32}$ Ainsworth, M. and Senior, B., "An adaptive refinement strategy for $h p$-finite element computations," Applied Numerical Mathematics, Vol. 26, 1998, pp. 165-178.

${ }^{33}$ Houston, P. and Süli, E., " $h p$-adaptive discontinuous Galerkin finite element methods for first-order hyperbolic problems," SIAM Journal on Scientific Computing, Vol. 23, No. 4, 2001, pp. 1226-1252.

${ }^{34}$ Demkowicz, L., Rachowicz, W., and Devloo, P., "A fully automatic hp-adaptivity," Journal of Scientific Computing, Vol. 17, 2002, pp. 117-142.

${ }^{35}$ Houston, P. and Süli, E., "A note on the design of hp-adaptive finite element methods for elliptic partial differential equations," Computer Methods in Applied Mechanics and Engineering, Vol. 194, 2005, pp. $229-243$.

${ }^{36}$ Fidkowski, K. J. and Darmofal, D. L., "An adaptive simplex cut-cell method for discontinuous Galerkin discretizations of the Navier-Stokes equations," AIAA Paper 2007-3941, 2007.

${ }^{37}$ Park, M. A., Anisotropic Output-Based Adaptation with Tetrahedral Cut Cells for Compressible Flows, Ph.D. thesis, Massachusetts Institute of Technology, Cambridge, Massachusetts, 2008.

${ }^{38}$ Carstensen, C. and Verfürth, R., "Edge residuals dominate a posteriori error estimates for low order finite element methods," SIAM Journal on Numerical Analysis, Vol. 36, No. 5, 1999, pp. 1571-1587.

${ }^{39}$ Houston, P., Rannacher, R., and Süli, E., "A posteriori error analysis for stabilised finite element approximations of transport problems," Computer Methods in Applied Mechanics and Engineering, Vol. 190, 2000, pp. 14831508.

${ }^{40}$ Berger, M. and Jameson, A., "Automatic adaptive grid refinement for the Euler equations." AIAA Journal, Vol. 23, 1985, pp. 561-568.

${ }^{41}$ Johansen, H. and Colella, P., "A Cartesian grid embedded boundary method for Poisson's equation on irregular domains," Journal of Computational Physics, Vol. 147, 1998, pp. 60-85.

${ }^{42}$ Sun, S. and Wheeler, M., "Mesh adaptation strategies for discontinuous Galerkin methods applied to reactive transport problems," International Conference on Computing, Communication and Control Techniques, H.W. Chu, M. Savoie, and B. Sanchez, Eds., 2004, pp. 223-228.

${ }^{43}$ Freitag, L. A. and Ollivier-Gooch, C., "Tetrahedral mesh improvement using swapping and smoothing," International Journal for Numerical Methods in Engineering, Vol. 40, 1997, pp. 3979-4002.

${ }^{44}$ Castro-Diaz, M. J., Hecht, F., Mohammadi, B., and Pironneau, O., "Anisotropic unstructured mesh adaptation for flow simulations," International Journal for Numerical Methods in Fluids, Vol. 25, 1997, pp. 475-491.

${ }^{45}$ Habashi, W. G., Dompierre, J., Bourgault, Y., Ait-Ali-Yahia, D., Fortin, M., and Vallet, M.-G., "Anisotropic mesh adaptation: towards user-independent, mesh-independent and solver-independent CFD. Part I: general principles," International Journal for Numerical Methods in Fluids, Vol. 32, 2000, pp. 725-744.

${ }^{46}$ Xia, G., Li, D., and Merkle, C. L., "Anisotropic grid adaptation on unstructured meshes," AIAA Paper 20010443, 2001.

${ }^{47}$ Baker, T. J., "Mesh adaptation strategies for problems in fluid dynamics," Finite Elements in Analysis and Design, Vol. 25, 1997, pp. 243-273.

${ }^{48}$ Park, M. A., "Adjoint-based, three-dimensional error prediction and grid adaptation," AIAA Paper 2002-3286, 2002. 2003.

${ }^{49}$ Park, M. A., "Three-dimensional turbulent RANS adjoint-based error correction," AIAA Paper 2003-3849,

${ }^{50}$ Shewchuk, J., "Delaunay refinement algorithms for triangular mesh generation," Computational Geometry: Theory and Applications, Vol. 22, 2002, pp. 21-74.

${ }^{51}$ Mavriplis, D. J., "Adaptive mesh generation for viscous flows using Delaunay triangulation," Journal of Computational Physics, Vol. 90, 1990, pp. 271-291.

${ }^{52}$ Borouchaki, H., George, P., Hecht, F., Laug, P., and Saltel, E., "Mailleur bidimensionnel de Delaunay gouverné par une carte de métriques. Partie I: Algorithmes," INRIA-Rocquencourt, France. Tech Report No. $2741,1995$.

${ }^{53}$ Hecht, F., "Bamg: Bidimensional anisotropic mesh generator," INRIA-Rocquencourt, France, 1998, http://pauillac.inria.fr/cdrom/www/bamg/bamg/eng.htm.

${ }^{54}$ Schall, E., Leservoisier, D., Dervieux, A., and Koobus, B., "Mesh adaptation as a tool for certified computational aerodynamics," International Journal for Numerical Methods in Fluids, Vol. 45, 2004, pp. $175-196$.

${ }^{55}$ Venditti, D. A. and Darmofal, D. L., "Anisotropic grid adaptation for functional outputs: application to two-dimensional viscous flows," Journal of Computational Physics, Vol. 187, No. 1, 2003, pp. 22-46.

${ }^{56}$ Aftosmis, M. and Berger, M., "Multilevel error estimation and adaptive h-refinement for Cartesian meshes with embedded boundaries," AIAA Paper 2002-14322, 2002.

${ }^{57}$ Nemec, M., Aftosmis, M. J., and Wintzer, M., "Adjoint-based adaptive mesh refinement for complex geometries," AIAA Paper 2008-0725, 2008.

${ }^{58}$ Becker, R. and Rannacher, R., "A feed-back approach to error control in finite element methods: Basic analysis and examples," East-West Journal of Numerical Mathematics, Vol. 4, 1996, pp. 237-264. 
${ }^{59}$ Nemec, M. and Aftosmis, M. J., "Error estimation and adpative refinement for embedded-boundary cartesian meshes," AIAA Paper 2007-4187, 2007.

${ }^{60}$ Zienkiewicz, O. C. and Zhu, J. Z., "Adaptivity and mesh generation," International Journal for Numerical Methods in Engineering, Vol. 32, 1991, pp. 783-810.

${ }^{61}$ Venditti, D. A., Grid Adaptation for Functional Outputs of Compressible Flow Simulations, Ph.D. thesis, Massachusetts Institute of Technology, Cambridge, Massachusetts, 2002.

${ }^{62}$ Peraire, J., Vahdati, M., Morgan, K., and Zienkiewicz, O. C., "Adaptive remeshing for compressible flow computations," Journal of Computational Physics, Vol. 72, 1987, pp. 449-466.

${ }^{63}$ Fidkowski, K. J. and Darmofal, D. L., "A triangular cut-cell adaptive method for highorder discretizations of the compressible navier-stokes equations," Journal of Computational Physics, Vol. 225, 2007, pp. 1653-1672.

${ }^{64}$ Formaggia, L., Micheletti, S., and Perotto, S., "Anisotropic mesh adaptation with applications to CFD problems," Fifth World Congress on Computational Mechanics, edited by H. A. Mang, F. G. Rammerstorfer, and J. Eberhardsteiner, Vienna, Austria, July 7-12 2002.

${ }^{65}$ Schneider, R. and Jimack, P. K., "Toward anisotropic mesh adaptation based upon sensitivity of a posteriori estimates," Tech. Rep. 2005.03, University of Leeds, School of Computing, 2005.

${ }^{66}$ Park, M. A. and Darmofal, D. L., "Parallel anisotropic tetrahedral adaptation," AIAA Paper 2008-917, 2008.

${ }^{67}$ Purvis, J. W. and Burkhalter, J. E., "Prediction of critical Mach number for store configurations," AIAA Journal, Vol. 17, No. 11, 1979, pp. 1170-1177.

${ }^{68}$ Wedan, B. and South, J. C., "A method for solving the transonic full-potential equation for general configurations," AIAA Paper 1983-1889, 1983.

${ }^{69}$ Clarke, D. K., Salas, M. D., and Hassan, H. A., "Euler calculations for multielement airfoils using Cartesian grids," AIAA Journal, Vol. 24, No. 3, 1986, pp. 353.

${ }^{70}$ De Zeeuw, D. and Powell, K. G., "An adaptively refined Cartesian mesh solver for the Euler equations," Journal of Computational Physics, Vol. 104, 1993, pp. 56-68.

${ }^{71}$ Coirier, W. J. and Powell, K. G., "Solution-adaptive cut-cell approach for viscous and inviscid flows," $A I A A$ Journal, Vol. 34, No. 5, 1996, pp. 938-945.

${ }^{72}$ Rubbert, P. E., Bussoletti, J. E., Johnson, F. T., Sidwell, K. W., Rowe, W. S., Samant, S. S., SenGupta, G., Weatherill, W. H., Burkhart, R. H., Everson, B. L., Young, D. P., and Woo, A. C., "A new approach to the solution of boundary value problems involving complex configurations," Computational Mechanics - Advances and Trends, edited by A. K. Noor, 1986, pp. 49-84.

${ }^{73}$ Young, D. P., Melvin, R. G., Bieterman, M. B., Johnson, F. T., Samant, S. S., and Bussoletti, J. E., "A higher-order boundary treatment for Cartesian-grid methods," Journal of Computational Physics, Vol. 92, 1991, pp. 1-66.

${ }^{74}$ Karman, S. L., "SPLITFLOW: A 3d unstructured Cartesian/prismatic grid CFD code for complex geometries," AIAA Paper 1995-0343, 1995.

${ }^{75}$ Löhner, R., Appanaboyina, S., and Cebral, J. R., "Comparison of body-fitted, embedded, and immersed solutions of low-Reynolds number 3-d incompressible flows," AIAA Paper 2007-1296, 2007.

${ }^{76}$ Aftosmis, M. J., "Solution adaptive Cartesian grid methods for aerodynamic flows with complex geometries," von Karman Institute for Fluid Dynamics, Lecture Series 1997-02, Rhode-Saint-Genése, Belgium, Mar. 3-7, 1997.

${ }^{77}$ Nemec, M., Aftosmis, M., Murman, S., and Pulliam, T., "Adjoint formulation for an embedded-boundary Cartesian method," AIAA Paper 2005-0877, 2005.

${ }^{78}$ Hunton, L., Hicks, R. M., and Mendoza, J. P., "Some effects of wing planform on sonic boom," NASA TN D-7160, 1973.

${ }^{79}$ Barter, G. and Darmofal, D., "Shock capturing with higher-order, PDE-based artificial viscosity." AIAA Paper 2007-3823, 2007.

${ }^{80}$ Gnoffo, P. A. and White, J. A., "Computational aerothermodynamic simulation issues for unstructured grids," AIAA Paper 2004-2371, 2004.

${ }^{81} \mathrm{Li}$, X., Shephard, M. S., and Beall, M. W., "Accounting for curved domains in mesh adaptation," International Journal for Numerical Methods in Engineering, Vol. 58, 2003, pp. 247-276.

${ }^{82} \mathrm{Si}, \mathrm{H}$., "Tetgen: A quality tetrahedral mesh generator and three-dimensional Delaunay triangulator," Weierstrass Institute for Applied Analysis and Stochastics, 2005, http://tetgen.berlios.de.

${ }^{83}$ Paraschivoiu, M., Peraire, J., and Patera, A., "A posteriori finite element bounds for linear functional outputs of elliptic partial differential equations," Computer Methods in Applied Mechanics and Engineering, Vol. 150, 1997, pp. 289-312.

${ }^{84}$ Peraire, J. and Patera, A., "Asymptotic a posteriori finite element bounds for the outputs of noncoercive problems: the helmholtz and burgers equation," Computer Methods in Applied Mechanics and Engineering, Vol. 171, 1999, pp. 77-86.

${ }^{85}$ Machiels, L., Peraire, J., and Patera, A., "A posteriori finite-element output bounds for the incompressible Navier-Stokes equations: Application to a natural convection problem," Journal of Computational Physics, Vol. 172, 
2001, pp. 401-425.

${ }^{86}$ Sauer-Budge, A. and Peraire, J., "Computing bounds for linear functionals of exact weak solutions to the advection-diffusion-reaction equation," Journal on Scientific Computing, Vol. 26, No. 2, 2004, pp. 636-652.

${ }^{87}$ Xuan, Z., Pares, N., and Peraire, J., "Computing upper and lower bounds for the J-integral in two-dimensional linear elasticity," Computer Methods in Applied Mechanics and Engineering, Vol. 195, 2006, pp. 430-443.

${ }^{88}$ Parés, N., Bonet, J., Huerta, A., and Peraire, J., "The computation of bounds for linear-functional outputs of weak solutions to the two-dimensional elasticity equations," Computer Methods in Applied Mechanics and Engineering, Vol. 195, 2006, pp. 406-429.

${ }^{89}$ Sarrate, J., Peraire, J., and Patera, A., "A posteriori finite element error bounds for nonlinear outputs of the Helmholtz equation," International Journal for Numerical Methods in Fluids, Vol. 31, 1999, pp. 17-36.

${ }^{90}$ Wong, J. and Peraire, J., "Implicit a-posteriori bounds for discontinuous Galerkin methods," AIAA Paper 2003-3844, 2003

${ }^{91}$ Lee, K.-H., Alonso, J. J., and van der Weide, E., "Mesh adaptation criteria for unsteady periodic flows using a discrete adjoint time-spectral formulation," AIAA Paper 2006-692, 2006.

${ }^{92}$ Nadarajah, S. K. and Jameson, A., "Optimum shape design for unsteady three-dimensional viscous flows using a nonlinear frequency-domain method," AIAA Journal of Aircraft, Vol. 44, No. 5, 2007, pp. 1513-1527.

${ }^{93}$ Nadarajah, S. K. and Jameson, A., "Optimum shape design for unsteady flows with time-accurate continuous and discrete adjoint methods," AIAA Journal, Vol. 45, No. 7, 2007, pp. 1478-1491.

${ }^{94}$ Nadarajah, S. K. and Jameson, A., "Optimal control of unsteady flows using a time accurate method," AIAA Paper 2002-5436, 2002.

${ }^{95}$ Rumpfkeil, M. P. and Zingg, D. W., "A general framework for the optimal control of unsteady flows with applications," AIAA Paper 2007-1128, 2007.

${ }^{96}$ Mani, K. and Mavriplis, D. J., "Discrete adjoint based time-step adaptation and error reduction in unsteady flow problems," AIAA Paper 2007-3944, 2007.

${ }^{97}$ Mani, K. and Mavriplis, D. J., "An unsteady discrete adjoint formulation for two-dimensional flow problems with deforming meshes," AIAA Paper 2007-60, 2007. 